EPJ manuscript No.

(will be inserted by the editor)

\title{
Attraction of like-charged macroions in the strong-coupling limit
}

\author{
Ali Naji ${ }^{a}$ and Roland R. Netz ${ }^{b}$ \\ ' Sektion Physik, Ludwig-Maximilians-Universität, Theresienstr. 37, D-80333 München, Germany. \\ ' Max-Planck-Institut für Kolloid- und Grenzflächenforschung, Am Mühlenberg, D-14476 Golm, Germany.
}

Ref.: Revised version published at Eur. Phys. J. E 13, 43 (2004).

\begin{abstract}
Like-charged macroions attract each other as a result of strong electrostatic correlations in the presence of multivalent counterions or at low temperatures. We investigate the effective electrostatic interaction between i) two like-charged rods and ii) two like-charged spheres using the recently introduced strong-coupling theory, which becomes asymptotically exact in the limit of large coupling parameter (i.e. for large counterion valency, low temperature, or high surface charge density on macroions). In contrast to previous applications of the strong-coupling theory, we deal with curved surfaces and an additional parameter, referred to as Manning parameter, is introduced, which measures the ratio between the radius of curvature of macroions to the Gouy-Chapman length. This parameter, together with the size of the confining box enclosing the two macroions and their neutralizing counterions, controls the counterion-condensation process that directly affects the effective interactions. For sufficiently large Manning parameters (weakly-curved surfaces), we find a strong long-ranged attraction between two macroions that form a closely-packed bound state with small surface-to-surface separation of the order of the counterion diameter in agreement with recent simulations results. For small Manning parameters (highly-curved surfaces), on the other hand, the equilibrium separation increases and the macroions unbind from each other as the confinement volume increases to infinity. This occurs via a continuous universal unbinding transition for two charged rods at a threshold Manning parameter of $\xi_{c}=2 / 3$, while the transition is strongly discontinuous for spheres because of a pronounced potential barrier at intermediate distances. Unlike the cylindrical case, the attractive forces between spheres disappear for increasing confinement volume due to the complete de-condensation of counterions. Scaling arguments suggest that for moderate values of coupling parameter, strong-coupling predictions remain valid for sufficiently small surface-to-surface separations.
\end{abstract}

PACS. 87.15.-v Biomolecules: structure and physical properties - 82.70.Dd Colloids - 87.15.Nn Properties of solutions; aggregation and crystallization of macromolecules

\section{Introduction}

Electrostatic interactions play a prominent role in many soft-matter and biological systems, since a huge class of macromolecules are water-soluble, and thus bear electric charges in aqueous solutions. These macroions may be polyelectrolytes such as DNA, or charged colloidal particles, that are surrounded by their neutralizing (oppositelycharged) counterions. In recent years, mounting evidence from both experiments [1,2,3,4,5,6] and numerical simu-

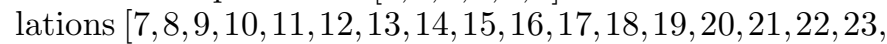
24.25 showed that like-charged macroions can attract each other via effective forces of electrostatic origin, notorious examples of which are formation of dense packages of DNA molecules (DNA condensates) [1] and large aggregates of charged colloidal particles [18, 19,20]. These observations indicate the appearance of like-charge attraction

\footnotetext{
a Present address: Physics Department, Technical University of Munich, 85748 Garching, Germany; e-mail: naji@ph.tum.de

b e-mail: netz@ph.tum.de
}

in strongly-charged systems, i.e. when multivalent counterions are present, macroions are highly charged, or the strength of electrostatic interactions is enhanced by maintaining the system at low temperatures or in a medium of low dielectric constant $[1,7,8,9,10,11,12,13,14,15,16,17$. [18, $19,20,21,22,23,24,25]$.

On the other hand, there has been a great deal of theoretical studies aimed at revealing possible underlying mechanisms, which can lead to such counter-intuitive interactions $6,8,8,9,10,11,12,13,14,15,16,17,18,19,20,21,22$.

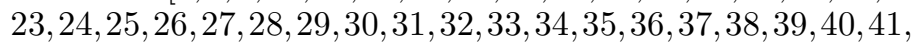

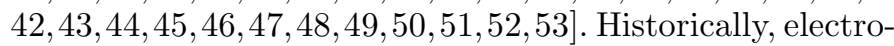
static interactions in charged systems have been studied by means of mean-field approximations, such as PoissonBoltzmann (PB) (or its linearized version, Debye-Hückel) theory [54 [55]. Despite the fact that in many instances, the PB theory provides a successful approximation, it has been shown rigorously [56 $57 \|, 58$ that such mean-field theories can only predict repulsion between like-charged objects. Recent investigations have, however, elucidated the 
Ali Naji, Roland R. Netz: Attraction of like-charged macroions in the strong-coupling limit

important role of electrostatic correlations, neglected in the mean-field approximation, toward understanding the mechanism of like-charge attraction. Several proposals have been put forward in order to incorporate ionic correlations. These attempts include integral-equation approach 26 . 27, perturbative improvement of the PB theory [28, 29, 30 , \begin{tabular}{ll|l|l|l|l|l|l|l|l}
31 & 32 & 33 & 34 & 35 & 36 & 37 & 38 & 39 & , local density-functional the-
\end{tabular} ory [40 41, structural-correlation approach [42,43.44, 45], harmonic-plasmon approximation 46, and the strong-coup theory 47 48 (that will be used in this paper), which generally compare well with simulation results and all exhibit attraction. Nonetheless, they are different in technical aspects, and have their own range of rigor and applicability.

In general, the importance of electrostatic correlations can be quantified by means of the coupling parameter $\Xi=2 \pi q^{3} \ell_{B}^{2} \sigma_{s}$ [4], which depends on the charge valency of counterions $q$, surface charge density of macroions $\sigma_{s}$, and the Bjerrum length $\ell_{B}=e^{2} /\left(4 \pi \varepsilon \varepsilon_{0} k_{B} T\right.$ ) (associated with a medium of dielectric constant $\varepsilon$ and at temperature $T$ ). The PB theory is asymptotically obtained in the limit of vanishingly small coupling parameter $\Xi \rightarrow 0$ [37, while, non-mean-field features emerge in the opposite limit of large coupling parameter, $\Xi \gg 1$, and are accompanied by strong accumulation of counterions in the proximity of charged objects, where counterions may also form strongly-correlated structures, such as a quasi-twodimensional liquid or possibly a Wigner crystal at low temperatures $16,19,42,45,46,48$.

For rod-like and spherical macroions, effective interactions are also influenced by the entropy-driven counterioncondensation process, which basically arises as a result of the specific interplay between energetic and entropic contributions in these geometries. Clearly, a dominant attraction between like-charged macroions necessitates a sufficiently large number of counterions being condensed in their close vicinity. The counterion-condensation process at rod-like macroions is controlled by the Manning parameter [59] defined here as $\xi=q \ell_{B} \tau$, where $\tau$ stands for the single-rod linear charge density. For small Manning parameter, counterions de-condense leading to a bare electrostatic repulsion between charged rods regardless of the coupling parameter. While for sufficiently large values of Manning parameter, an attractive force may prevail as a certain fraction of counterions remains bound to the rods even in the absence of confining boundaries. An interesting problem is, therefore, to examine the regime of Manning parameters, where an effective attraction emerges. This has been addressed in a number of previous works for a pair of like-charged rods. It was argued by Ray and Manning 49] that attraction sets in, when the Manning parameter becomes larger than the threshold of counterion condensation in the two-rod system, i.e. $\xi=1 / 2$. While, a different attraction range of $\xi>2$ was proposed by the counterion-condensation theory of Arenzon et al. 44. Numerical simulations, on the other hand, reveal attraction between two like-charged rods in a wide range of Manning parameters including $\xi \approx 1$ (for moderate coupling parameters) [13 23 24 25], though have not yet specified the threshold value.
For a system of charged spheres, attraction is induced only when the system is confined in a box, because the energetic binding of counterions to charged spheres is weaker than the entropic gain resulting in complete de-condensation of counterions in the absence of confining boundaries. The influence of the confinement volume on the effective interaction between like-charged spheres was addressed in the simulations by Grønbech-Jensen et al. 14. It was shown intgat the pair potential of mean force develops a local minimum at small surface-to-surface separation, which corresponds to a closely-packed bound state of spheres maintained by a strong short-ranged attraction, as indicated independently by other simulations $[15,16$. This attraction regime is separated from a repulsion regime at large separations by a pronounced potential barrier 14. Upon increasing the confinement volume, the strength of attraction and the barrier height decrease leading to pure repulsion between spheres in a very large confining box. Other simulations on many-sphere systems [18, 19, have also displayed formation of large aggregates of highly-charged spheres, which indicate a phase separation in the thermodynamic limit [1, 18,19]. As discussed by Linse et al. [19, the equilibrium surface-to-surface separation of the attracting spheres in the compact state appears to be of the order of the counterion diameter.

In this paper, we investigate the effective interaction between a pair of like-charged rods and a pair of likecharged spheres using the strong-coupling (SC) theory introduced in previous works [7]. In contrast to previous applications of the strong-coupling theory [47,48], we deal with curved surfaces and thus, an additional parameter is introduced, which measures the ratio between the radius of curvature of macroions to the associated GouyChapman length. For charged rods, this parameter is equivalent with the so-called Manning parameter (introduced above), and thus, we shall refer to this additional parameter as Manning parameter for both charged rods and charged spheres. We shall identify the regime of Manning parameters, where attraction is predicted between macroions with or without any confinement, and examine the equilibrium separation of interacting macroions for varying Manning parameter and confinement volume.

The SC theory is obtained by a systematic virial expansion in the limit of large coupling parameter, $\Xi \rightarrow \infty$, which is complementary to the mean-field regime (Section 2). This, in particular, covers the low-temperature regime (but still above the crystallization temperature 48] . Nonetheless, the theory involves finite entropic contributions, thus finite-temperature effects from counterions that appear at leading order in a simple one-particle form. This allows for reproducing the de-condensation process of counterions at low Manning parameters and therefore, leads to a consistent picture in the whole range of Manning parameters. For sufficiently large Manning parameters, when counterions effectively condense around macroions, we find a long-ranged attraction, which originates mainly from the energetic attraction mediated by counterions intervening in a narrow region between macroions. The effective force is dominated by such an attraction 
for increasing Manning parameter (or decreasing temperature) and tends to a temperature-independent value, which scales with the macroion separation, $D$, as $\sim 1 / D$ for two charged rods, and as $\sim 1 / D^{2}$ for two charged spheres. (For two charged plates, the effective force is obtained to be independent of $D$ 47 48.) In this regime, two macroions are predicted to form a closely-packed bound state with a surface-to-surface distance of the order of the counterion diameter in qualitative agreement with numerical findings 14, 15, 18, 19, 23, 24. Such an agreement is established, when the excluded-volume repulsion between counterions is negligible compared to the electrostatic interactions. For point-like counterions, the equilibrium surfaceto-surface distance is predicted to be of the order of the Gouy-Chapman length - see Eqs. (39) and (58) below- and a quantitative agreement is obtained between SC predictions and simulation results as reported elsewhere [25].

We shall also study the influence of the confinement due to an outer box on the effective interaction and on the onset of attraction. It will be shown that by decreasing the Manning parameter or increasing the size of the confinement, macroions undergo an unbinding transition. For two charged rods, the minimal Manning parameter to obtain attraction (the threshold Manning parameter) tends to the universal value of $\xi_{c}=2 / 3$ for increasing box size, which is somewhat larger than the corresponding onset of counterion condensation $\xi=1 / 2$. The unbinding transition in this system occurs continuously and is characterized by a power-law behavior. In contrast, charged spheres display a discontinuous transition and a potential barrier in the effective free energy in qualitative agreement with simulation results [14. It turns out that the attraction threshold for two like-charged spheres monotonically increases with the size of the confining box yielding a pure repulsion between unconfined spheres, but its dependence on the box size is logarithmically weak. Such a weak dependence of the onset of attraction on the box size may explain the stability of aggregates of highly-charged spheres in large confinements that has been observed in recent simulations [18, 19, 20]. The range of applicability of the present theory to systems with finite coupling parameter will be examined by means of scaling arguments.

\section{Strong-Coupling Theory: General formalism}

Consider a system of macroions with charge distribution $\sigma(\mathbf{r})$ (in units of the elementary charge $e$ ), and a number of $N$ oppositely-charged counterions of diameter $\sigma_{c}$ and charge valency $q$. All charges interact via Coulombic interaction $v(\mathbf{r})=1 /|\mathbf{r}|$, and the electroneutrality condition is assumed to hold globally in the system. Hence, one always has

$$
N q=\int d \mathbf{r} \sigma(\mathbf{r})
$$

Note that $q$ and $\sigma(\mathbf{r})$ are defined to be positive. We shall suppose that macroions are fixed. (In the forthcoming calculations in Sections 3 and 4 we shall consider excludedvolume interactions of hard-core nature between particles- see Section 2.1) Assuming a surface charge density of $\sigma_{s}$ for macroions, the Gouy-Chapman length, $\mu$, is defined as

$$
\mu=\frac{1}{2 \pi q \ell_{B} \sigma_{s}}
$$

which sets a characteristic length scale for the considered system. Here, $\ell_{B}=e^{2} /\left(4 \pi \varepsilon \varepsilon_{0} k_{B} T\right)$ is the Bjerrum length, the distance at which two elementary charges interact with thermal energy $k_{B} T$ in a medium of dielectric constant $\varepsilon$.

It is convenient to construct a dimensionless formalism, which may be achieved by rescaling spatial coordinates, say $\mathbf{r}$, with the Gouy-Chapman length as $\tilde{\mathbf{r}}=\mathbf{r} / \mu$. Other parameters are rescaled accordingly, for instance,

$$
\tilde{\sigma}(\tilde{\mathbf{r}})=\mu \sigma(\mathbf{r}) / \sigma_{s}
$$

In the rescaled units and for point-like counterions $\left(\sigma_{c}=\right.$ 0 ), the grand-canonical partition function of the system, $\mathcal{Z}_{\Lambda}$, is mapped to a one-parametric field theory (apart from rescaled geometrical parameters such as the typical macroion size), and may be expressed as a functional integral over a fluctuating field $\phi(\tilde{\mathbf{r}})$ 47]

$$
\mathcal{Z}_{\Lambda}=\int \frac{\mathcal{D} \phi}{\mathcal{Z}_{v}} \exp \{-\tilde{\mathcal{H}}[\phi] / \Xi\}
$$

where

$$
\Xi=2 \pi q^{3} \ell_{B}^{2} \sigma_{s}
$$

is the coupling parameter, $\mathcal{Z}_{v}=\sqrt{\operatorname{Det} v}$ contains selfenergy contributions and the action reads

$\tilde{\mathcal{H}}[\phi]=\frac{1}{2 \pi} \int d \tilde{\mathbf{r}}\left[\frac{1}{4}(\nabla \phi(\tilde{\mathbf{r}}))^{2}-\imath \phi(\tilde{\mathbf{r}}) \tilde{\sigma}(\tilde{\mathbf{r}})-\Lambda \tilde{\Omega}(\tilde{\mathbf{r}}) e^{-\imath \phi(\tilde{\mathbf{r}})}\right]$.

Here, $\Lambda$ is the rescaled fugacity, and the function $\tilde{\Omega}(\tilde{\mathbf{r}})$ takes geometrical constraints into consideration, e.g. restricts the positions of mobile counterions to an appropriate region in space. The electroneutrality condition, Eq. (11), written in rescaled units, relates the coupling parameter to the average number of counterions through

$$
\tilde{Q} \equiv \int d \tilde{\mathbf{r}} \tilde{\sigma}(\tilde{\mathbf{r}})=2 \pi \Xi N,
$$

where $\tilde{Q}$ is simply the rescaled area of macroions covered by electric charges.

In general, statistical quantities such as counterions density profile, osmotic pressures and effective forces can be obtained from Eq. (4). But, as exact calculations based on the partition function (44) are difficult, one may consider limiting cases, where approximate methods are applicable. For instance, at small coupling parameters $\Xi \rightarrow 0$, a saddle-point approximation may be applied, since the functional integral in this limit is dominated by the saddle point of the action, Eq. (6). This approximation yields the so-called mean-field Poisson-Boltzmann (PB) theory, which may be then extended to finite couplings by means of a systematic loop expansion around the saddle-point solution [37. However, the loop expansion around PB theory was shown to be a weakly convergent series and can 
not improve the PB results to be applicable for systems of large coupling parameter 48 .

At large coupling parameters $\Xi \gg 1$, a series expansion can be obtained for the partition function (4) in powers of the rescaled fugacity, $\Lambda / \Xi$, as 47 ]

$$
\begin{aligned}
\mathcal{Z}_{\Lambda} & =\mathcal{Z}_{0} \sum_{j=0}^{\infty} \frac{1}{j !}\left[\frac{\Lambda}{2 \pi \Xi}\right]^{j} \prod_{k=1}^{j}\left[\int d \tilde{\mathbf{r}}_{k} \tilde{\Omega}\left(\tilde{\mathbf{r}}_{k}\right)\right] \\
& \times \exp \left\{-\Xi \sum_{n<m}^{j} v\left(\tilde{\mathbf{r}}_{n}-\tilde{\mathbf{r}}_{m}\right)-\sum_{i=1}^{j} \tilde{u}\left(\tilde{\mathbf{r}}_{i}\right)\right\},
\end{aligned}
$$

which is nothing but a virial expansion with respect to the counterionic degrees of freedom. The zeroth-order term of the expansion, $\mathcal{Z}_{0}$, represents the partition function of the system, when all counterions are taken out and only the fixed charge distribution is present,

$$
\mathcal{Z}_{0}=e^{-\tilde{U}_{0} / \pi \Xi}
$$

where

$\tilde{U}_{0}=\frac{1}{8 \pi} \int d \tilde{\mathbf{r}} d \tilde{\mathbf{r}}^{\prime} \tilde{\sigma}(\tilde{\mathbf{r}}) v\left(\tilde{\mathbf{r}}-\tilde{\mathbf{r}}^{\prime}\right) \tilde{\sigma}\left(\tilde{\mathbf{r}}^{\prime}\right)-\frac{\tilde{Q}}{4 \pi} \int d \tilde{\mathbf{r}}^{\prime} v\left(\tilde{\mathbf{r}}^{\prime}-\tilde{\mathbf{r}}_{0}\right) \tilde{\sigma}\left(\tilde{\mathbf{r}}^{\prime}\right)$

is the rescaled zero-particle interaction energy. (Note that $\tilde{\mathbf{r}}_{0}$ is an arbitrary point chosen to fix the reference configuration with respect to which the interaction energy is calculated.)

The first-order term in Eq. (8) is the partition function of the system in the presence of a single counterion interacting with the fixed charge distribution via the oneparticle interaction

$$
\tilde{u}(\tilde{\mathbf{r}})=-\frac{1}{2 \pi} \int d \tilde{\mathbf{r}}^{\prime}\left[v\left(\tilde{\mathbf{r}}-\tilde{\mathbf{r}}^{\prime}\right)-v\left(\tilde{\mathbf{r}}_{0}-\tilde{\mathbf{r}}^{\prime}\right)\right] \tilde{\sigma}\left(\tilde{\mathbf{r}}^{\prime}\right)
$$

Higher-order terms in Eq. (8) involve the two-particle interaction $v\left(\tilde{\mathbf{r}}-\tilde{\mathbf{r}}^{\prime}\right)$, that emerges in the non-perturbative form of $\exp (-\Xi v)$.

The canonical strong-coupling (SC) theory is obtained as an asymptotic theory from the above expansion, Eq. (87), in the limit of $\Xi \rightarrow \infty$. As we shall see below (Section 2.1), the leading-order term in the canonical free energy contains counterionic contributions only up to the one-particle terms. This reflects the fact that for large $\Xi$, counterion-macroion correlations dominate over counterioncounterion correlations. Higher-order corrections for finite couplings have been studied analytically for systems composed of planar charged walls in Refs. [47,48] and will not be considered in the present study.

Clearly, the virial expansion, Eq. (8), could have been obtained directly from the original partition function of the system. Also note that although the field-theoretic representation of the grand-canonical partition function, Eq. (44), was obtained for point-like counterions, the virial expansion, Eq. (8), is quite general and can systematically include finite counterion size $\left(\sigma_{c} \neq 0\right)$ via excluded-volume interactions between particles (see Section 2.1). Taking the detour over the field-theoretic formulation based on Eqs.
(4) and (6), however, demonstrates that loop expansion and virial expansion are indeed the two asymptotic limits of the same problem: While the PB theory and loop expansion become asymptotically exact in the limit of weak coupling $\Xi \rightarrow 0$, the SC theory and the virial expansion produce asymptotically exact results in the complementary limit of large coupling $\Xi \rightarrow \infty$. One may notice that higher-order terms in Eq. (8) contain integrations over the so-called Mayer function $\exp (-\Xi v)-1$, that are weighted by other factors coming from the interaction of counterions with macroions. Such integrations are known to be divergent for bulk systems such as electrolytes, where the virial expansion fails to produce a convergent low-density expansion for the equation of state. In contrast, here counterions are considered at charged macroscopic objects. It has been shown explicitly for charged planar walls that in the limit of $\Xi \rightarrow \infty$, all orders of the virial expansion produce finite contributions to the density profile of counterions and the effective force between walls [4].

\subsection{The strong-coupling free energy}

To calculate the strong-coupling free energy, we start from the grand-canonical free energy (in units of $k_{B} T$ ),

$$
\mathcal{Q}_{\Lambda}=-\ln \mathcal{Z}_{\Lambda}
$$

where $Z_{\Lambda}$ is given by Eq. (8). The Legendre transformation

$$
\mathcal{F}_{N}=N \ln \Lambda+\mathcal{Q}_{\Lambda},
$$

provides us with the canonical free energy $\mathcal{F}_{N}$. The rescaled fugacity, $\Lambda$, in Eq. (13) is calculated from

$$
N=\Lambda \frac{\partial \ln \mathcal{Z}_{\Lambda}}{\partial \Lambda}
$$

in terms of $\Xi$ and other rescaled geometrical factors. (Note that $N$ can be eliminated using Eq. (7).) In general, we may propose the following expression for $\Lambda$ in the large coupling limit $\Xi \gg 1$,

$$
\Lambda=\Lambda_{0}+\frac{\Lambda_{1}}{\Xi}+\frac{\Lambda_{2}}{\Xi^{2}}+\ldots,
$$

where $\Lambda_{0}, \Lambda_{1}, \ldots$ are determined from Eq. (14) using Eqs. (7), (8)-(11) 61]. It is easy to verify that, for instance,

$$
\begin{aligned}
& \Lambda_{0}=\frac{\tilde{Q}}{\int d \tilde{\mathbf{r}} \tilde{\Omega}(\tilde{\mathbf{r}}) e^{-\tilde{u}(\tilde{\mathbf{r}})}} \\
& \Lambda_{1}=\frac{\tilde{Q}^{2} \int d \tilde{\mathbf{r}} d \tilde{\mathbf{r}}^{\prime} \tilde{\Omega}(\tilde{\mathbf{r}}) \tilde{\Omega}\left(\tilde{\mathbf{r}}^{\prime}\right) e^{-\tilde{u}(\tilde{\mathbf{r}})-\tilde{u}\left(\tilde{\mathbf{r}}^{\prime}\right)}\left[1-e^{-\Xi v\left(\tilde{\mathbf{r}}-\tilde{\mathbf{r}}^{\prime}\right)}\right.}{2 \pi\left[\int d \tilde{\mathbf{r}} \tilde{\Omega}(\tilde{\mathbf{r}}) e^{-\tilde{u}(\tilde{\mathbf{r}})}\right]^{3}}
\end{aligned}
$$

Inserting this into Eq. (13), we find the canonical free energy $\mathcal{F}_{N}$ (in units of $k_{B} T$ and up to an irrelevant additive constant), which also admits a large-coupling expression

$$
\mathcal{F}_{N}=\frac{\mathcal{F}_{1}}{\Xi}+\frac{\mathcal{F}_{2}}{\Xi^{2}}+\ldots
$$


where the coefficient of the leading-order term is

$$
\mathcal{F}_{1}=\frac{\tilde{U}_{0}}{\pi}-\frac{\tilde{Q}}{2 \pi} \ln \int d \tilde{\mathbf{r}} \tilde{\Omega}(\tilde{\mathbf{r}}) e^{-\tilde{u}(\tilde{\mathbf{r}})}+C_{0}
$$

in which $C_{0}=(\tilde{Q} / 2 \pi) \ln \tilde{Q}-\tilde{Q} / 2 \pi$ is a constant, and the geometry function $\tilde{\Omega}$, as introduced before, specifies the accessible volume for counterions.

The free energy coefficient $\mathcal{F}_{1}$, which we may refer to as the rescaled $S C$ free energy of the system, yields the actual strong-coupling free energy, $\mathcal{F}_{N}^{S C}$, that is

$$
\mathcal{F}_{N}^{S C}=\frac{\mathcal{F}_{1}}{\Xi}
$$

which generates the leading (non-vanishing) contribution to the effective forces between macroions 62. This will be used in the following Sections to investigate the effective interaction between two charged rods and two charged spheres in the strong-coupling limit. The SC free energy has a non-trivial temperature dependence that will be discussed briefly in the Appendix for some asymptotic cases.

For realistic systems with finite coupling parameter, higher-order corrections to the SC free energy become increasingly important as in this situation, counterioncounterion correlations become comparable to the counterionmacroion correlations accounted for by the asymptotic term. Thus the range of applicability of SC predictions at moderate to large couplings should be examined by calculating higher-order corrections. These calculations are, however, difficult to perform analytically for the system of two charged rods and two charged spheres considered in this paper. Thus, we shall use qualitative schemes to identify the regime of validity of SC results based on the fact that electrostatic correlations induced by counterions between apposing macroions are dominant, when the typical lateral distance between counterions becomes larger than or comparable to the macroions surface-to-surface separation. This criterion, which was first introduced by Rouzina and Bloomfield [42, has been derived by calculating higher-order terms in Eq. (8) for planar charged walls 47, and is supported by numerical simulations [48]. This has also been addressed in simulations on charged rods and charged spheres in Refs. [16, 18, 19, 25].

Finally, as discussed before, specific excluded-volume interactions between particles can be accounted for systematically within the virial expansion, Eq. (8), which allows for treating counterions of finite diameter, $\sigma_{c}$, within the strong-coupling scheme. However, as clearly seen from Eq. (19), only macroion-counterion excluded-volume interaction enters in the leading-order contribution to the free energy in the SC limit. The excluded-volume interaction between counterions themselves appears only in the higher-order terms, which are not considered here. For the sake of simplicity in the forthcoming calculations, we shall assume a hard-core repulsion between counterions and macroions, therefore, overlapping configurations do not contribute to the spatial integral in Eq. (19). In other words, the effect of counterion size in the SC limit is incorporated into the geometry function $\tilde{\Omega}$, which, for

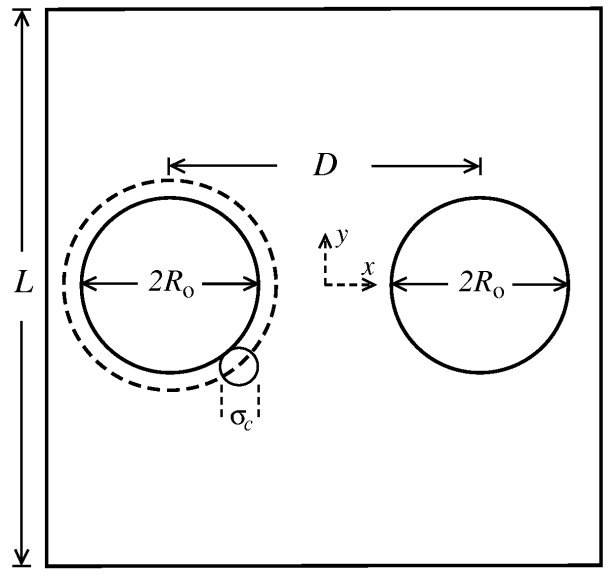

Fig. 1. The geometry of the system of two similar rods confined in a rectangular box (top view) as considered in the text (Section 3). Dashed circle shows the closest approach distance between macroions of radius $R_{0}$ and counterions of diameter $\sigma_{c}$ (see Eq. (21)).

cylindrical and spherical macroions of radius $R_{0}$, implies a hard-core radius of

$$
R=R_{0}+\sigma_{c} / 2
$$

Now, for a given amount of macroion charge, Equation (21) implies a reduced surface charge density, and thus an increased Gouy-Chapman length, Eq. (2), as compared to the case with point-like counterions. The following results are, however, presented in units of the Gouy-Chapman length and the counterion diameter explicitly appears only when the actual units are restored (see e.g. Eqs. (39) and (58) below).

\section{Two Like-Charged Rods}

Consider two similar and infinitely-long rods each of radius $R_{0}$, length $H$ and the linear charge density $\tau$ (in unit of the elementary charge $e$ ). We assume that the axes of the rods are parallel and located at separation $D$ from each other. (We choose the frame of coordinates such that its origin lies in the mid-way between the rods and its $z$-axis is parallel to the rods axes - see Figure 1) To consider a more general case, we suppose that in lateral directions, the system is confined in a rectangular box with the edge size of $L$. The electroneutrality condition is fulfilled globally due to the presence of oppositely-charged counterions of valency $q$ inside the box. Assuming that the electric charge is distributed uniformly over the surface of the rods, their surface charge density reads $\sigma_{s}=\tau /(2 \pi R)$, and the Gouy-Chapman length, Eq. (2), is

$$
\mu=\frac{R}{\ell_{B} q \tau},
$$

where $R$ is the hard-core radius defined according to Eq. (21). As it follows from Eq. (22), the rescaled rod radius, 
$\tilde{R}=R / \mu$, is identical with the Manning parameter, i.e.

$$
\tilde{R}=\xi=\ell_{B} q \tau .
$$

The rescaled SC free energy of the two-rod system, $\mathcal{F}_{1}$, follows from Eq. (19), in which the zero-particle interaction energy, $\tilde{U}_{0}$, and the one-particle interaction energy, $\tilde{u}$, are obtained from Eqs. (10) and (11) respectively. Neglecting irrelevant self-energy terms and taking the off-set point $\mathbf{r}_{0}=\mathbf{0}$, we have

$$
\begin{aligned}
\frac{\tilde{U}_{0}}{\tilde{H}} & =6 \pi \tilde{R}^{2} \ln \tilde{D}, \\
\tilde{u}(\tilde{x}, \tilde{y}) & =2 \tilde{R}\left[\ln \tilde{r}_{1}+\ln \tilde{r}_{2}-\ln \left(\tilde{D}^{2} / 4\right)\right],
\end{aligned}
$$

where

$$
\begin{aligned}
& \tilde{r}_{1}=\left[(\tilde{x}+\tilde{D} / 2)^{2}+\tilde{y}^{2}\right]^{1 / 2}, \\
& \tilde{r}_{2}=\left[(\tilde{x}-\tilde{D} / 2)^{2}+\tilde{y}^{2}\right]^{1 / 2},
\end{aligned}
$$

are radial distances from the rods axes. Inserting the above expressions into Eq. (19), we end up with the SC free energy (per unit length of the rods, $\tilde{H}$, and up to an irrelevant additive term) as

$$
\frac{\mathcal{F}_{1}}{\tilde{H}}=-2 \tilde{R}^{2} \ln \tilde{D}-2 \tilde{R} \ln I
$$

where

$$
I(\tilde{D}, \tilde{R}, \tilde{L}) \equiv \int d \tilde{x} d \tilde{y} \tilde{\Omega} e^{-2 \tilde{R}\left[\ln \tilde{r}_{1}+\ln \tilde{r}_{2}\right]}
$$

The geometry function $\tilde{\Omega}=\tilde{\Omega}(\tilde{x}, \tilde{y} ; \tilde{D}, \tilde{R}, \tilde{L})$ specifies the region of integration, i.e. it is one inside the volume surrounded by the rectangular box and the cylindrical rods, and is zero elsewhere.

\subsection{Threshold of attraction}

The first term in the rescaled SC free energy, Eq. (27), simply gives the long-ranged (bare) electrostatic repulsion between the rods, and the second term involving the spatial integral contains energetic and entropic contributions of counterions on the leading order, which can generate an effective rod-rod attraction. In order to examine the counterionic contribution, and the onset of attraction, let us consider first the limit of two unconfined rods, i.e. when the box size becomes infinitely larger than the rod radius, $\tilde{L} / \tilde{R} \rightarrow \infty$. In this limit, the counterionic integral $I$, Eq. (28), diverges for Manning parameters $\tilde{R}<1 / 2$, which can be seen by rescaling the spatial coordinates with the box size according to $\tilde{x} \rightarrow \tilde{x} / \tilde{L}$, etc., that yields $I \sim \tilde{L}^{2-4 \tilde{R}}$. This indicates that $\tilde{R}=1 / 2$ is the threshold of counterion condensation in this system, below which the distribution function of counterions around the rods vanishes 63 in agreement with the value obtained from Manning condensation theory [49]. Moreover, the counterion-mediated force,

$$
\tilde{F}_{c i} \sim \frac{\partial}{\partial \tilde{D}} \ln I
$$

vanishes for $\tilde{R}<1 / 2$ as the box size tends to infinity. Thus, attraction between unconfined rods can only set in for Manning parameters larger than $1 / 2$. Now assuming that the SC free energy has only one local minimum, which is indeed the case as we shall see from the numerical solution, the onset of attraction can be determined by examining the large-separation behavior of the free energy, i.e. for $\tilde{D} \gg \tilde{D}_{\min }$, where $\tilde{D}_{\min }=2 \tilde{R}$ is the smallest possible axial separation of the rods. To this end, we rescale the spatial coordinates with the axial separation $\tilde{D}$ as

$$
\tilde{x}^{\prime}=\frac{\tilde{x}}{\tilde{D}}, \quad \tilde{y}^{\prime}=\frac{\tilde{y}}{\tilde{D}} .
$$

Accordingly, the integral $I$ in Eq. (28) scales as

$$
I(\tilde{D}, \tilde{R})=\tilde{D}^{2-4 \tilde{R}} J\left(\frac{\tilde{R}}{\tilde{D}}, \tilde{R}\right),
$$

where $J$ is a dimensionless integral given by

$J=2 \int_{\tilde{x}^{\prime}>0} d \tilde{x}^{\prime} d \tilde{y}^{\prime}\left[\left(\tilde{x}^{\prime}+\frac{1}{2}\right)^{2}+\left(\tilde{y}^{\prime}\right)^{2}\right]^{-\tilde{R}}\left[\left(\tilde{x}^{\prime}-\frac{1}{2}\right)^{2}+\left(\tilde{y}^{\prime}\right)^{2}\right]^{-\tilde{R}} \tilde{\Omega}^{\prime}$,

where we have made use of the symmetry property of the integrand upon the reflection with respect to the plane $\tilde{x}^{\prime}=0$, and thus the corresponding integral runs over the half-space $\tilde{x}^{\prime}>0$ excluding a disk of radius $\tilde{R} / \tilde{D}$ centered at $\left(\tilde{x}^{\prime}=+1 / 2, \tilde{y}^{\prime}=0\right.$ ) (this is formally accounted for by the geometry function $\left.\tilde{\Omega}^{\prime}\right)$. For very large $\tilde{D} / \tilde{D}_{\text {min }}$, the radius of the disk tends to zero and the limiting behavior of the integral in Eq. (32) is determined by the contributions from the boundary regions, which vary depending on whether $\tilde{R}$ is smaller or larger than 1 .

For Manning parameter $\tilde{R}<1$, the contribution of the boundary region around the disk vanishes, and the integral in Eq. (32) is dominated by its outer boundary, which gives only a constant independent of $\tilde{D}$. Therefore, the prefactor of $J$ in Eq. (31) yields the leading $\tilde{D}$-dependence of $I$ for large axial separations, and substituting this into Eq. (27) gives the limiting form of the rescaled SC free energy as

$$
\frac{\mathcal{F}_{1}}{\tilde{H}} \approx-2 \tilde{R}(2-3 \tilde{R}) \ln \tilde{D} .
$$

For $\tilde{R}>1$, on the other hand, the integral in Eq. (32) is dominated by the boundary region around the disk yielding $J \sim \tilde{D}^{2 \tilde{R}-2}$ for very large $\tilde{D} / \tilde{D}_{\text {min }}$, which leads to the following attractive tail for the rescaled SC free energy,

$$
\frac{\mathcal{F}_{1}}{\tilde{H}} \approx 2 \tilde{R}^{2} \ln \tilde{D} .
$$

Therefore, as clearly seen from Eqs. (33) and (34), two unconfined rods experience a repulsive force at large separations, when Manning parameter, $\tilde{R}$, becomes smaller than the threshold

$$
\tilde{R}_{c}=\frac{2}{3},
$$

and on the contrary, they attract each other for larger Manning parameters $\tilde{R}>\tilde{R}_{c}$. This result is obtained also by numerical calculation of the free energy (Section 3.2). 


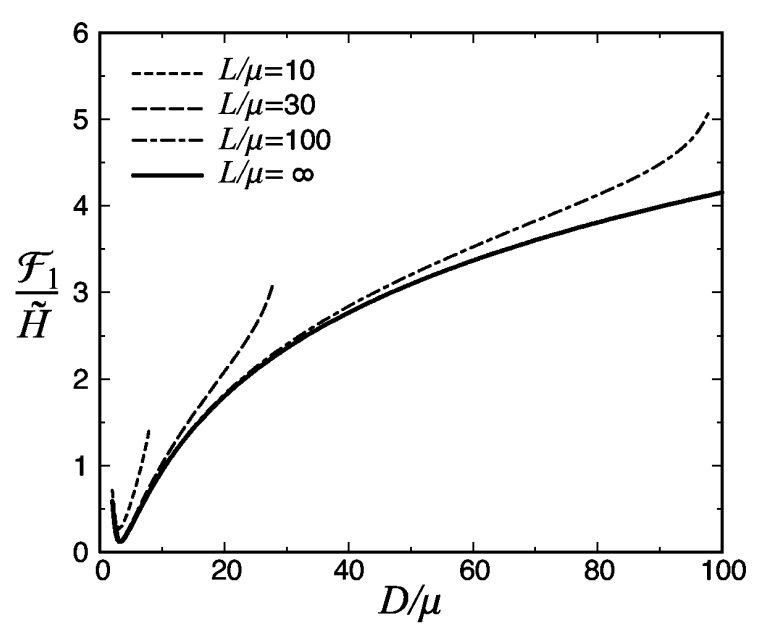

Fig. 2. The rescaled strong-coupling free energy of the two-rod system, Eq. (27), as a function of the rescaled axial separation, $\tilde{D}=D / \mu$, for Manning parameter $\tilde{R}=1.0$ and different box sizes indicated on the graph.

The result that the onset of attraction for two unconfined rods is predicted to be larger than the threshold of the counterion condensation $(\tilde{R}=1 / 2)$ could be anticipated from the beginning, since right at the threshold of the condensation, there remains an unbalanced bare repulsion between the rods according to the SC free energy, Eq. (27). The onset of attraction has also been considered in a number of recent studies. Analysis of Ray and Manning 49 based on the standard counterion-condensation model predicts attraction for $\tilde{R}>1 / 2$. In contrast, results from the counterion-condensation theory of Arenzon et al. 44 suggest a larger value of $\tilde{R}=2$ for the threshold of attraction. Numerical simulations, on the other hand, indicate the appearance of attraction for a wide range of Manning parameters including $\tilde{R} \approx 1$ [13, 23, 24, 25]. The precise location of the threshold of attraction between two charged rods is currently being studied by means of MD simulations 24,25].

The attractive force predicted in the strong-coupling limit is long-ranged and scales inversely with the axial separation between the rods (see Eqs. (33) and (34) and Figure 2). It should be noted that the SC attraction originates mainly from the energetic interaction mediated by counterions sandwiched between the rods, and in this respect, is different from the attraction obtained by the Gaussianfluctuation theories 28 31,33,34. Specifically, for increasing Manning parameter (or decreasing temperature), the strength of SC attraction increases and saturates to a temperature-independent value (see Appendix A.1). This can be seen also from the large-separation behavior of the free energy for highly-charged rods, Eq. (34), which gives the attractive force as

$$
\frac{F_{\text {rods }}}{H} \approx-\frac{e^{2} \tau^{2}}{2 \pi \varepsilon \varepsilon_{0} D}
$$

in actual units, where $F_{\text {rods }}$ is obtained from the actual SC free energy, Eq. (20), as $F_{\text {rods }}=-\left(k_{B} T\right) \partial \mathcal{F}_{N}^{S C} / \partial D$. Such

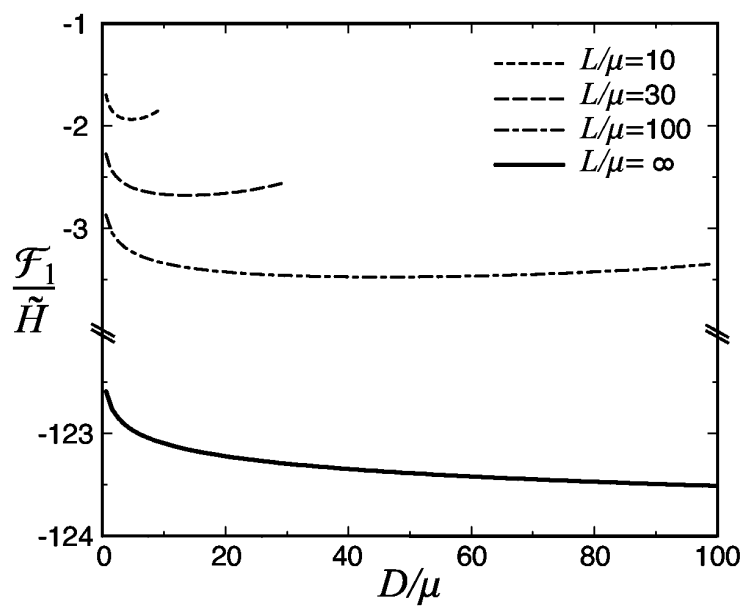

Fig. 3. The same as Figure 2 but for Manning parameter $\tilde{R}=0.3$.

an energetic attraction is also obtained for planar charged walls 47,48 and charged spheres (Section 4), but with different dependencies on $D$. The mechanism of SC attraction for large $\tilde{R}$, therefore, qualitatively agrees with the low-temperature results 44, 45] (see also Refs. 34, 38, 46] for discussions on the crossover from the low-temperature to the high-temperature regime).

\subsection{Equilibrium axial distance}

Now, let us consider a more general case with a rectangular box of edge size $L$ (Figure 1). For sufficiently large Manning parameters $\tilde{R}>\tilde{R}_{c}=2 / 3$, the presence of confining walls is expected to have only a minute effect on the effective rod-rod interaction since most of the counterions are strongly localized in the proximity of the rods. Figure 2 shows the rescaled SC free energy of the two-rod system, calculated numerically from Eq. (27), as a function of the axial separation, $\tilde{D}$, for Manning parameter $\tilde{R}=1$ and various box sizes. As seen, the free energy quickly converges to the free energy of an unconfined system with $\tilde{L}=\infty$, and the long-ranged attraction is only slightly strengthened at large separations, when rods are located close to the box boundaries. The free energy takes its minimum at a small axial distance, $\tilde{D}_{*} \approx 2 \tilde{R}$, which corresponds to a closely-packed bound state of rods. (We may refer to $\tilde{D}_{*}$ as rescaled "equilibrium" axial separation.) The approximate form of $\mathcal{F}_{1}$ in the vicinity of its minimum can be calculated as

$$
\frac{\mathcal{F}_{1}}{\tilde{H}} \approx 6 \tilde{R}^{2} \ln \tilde{D}-2 \tilde{R} \ln (\tilde{D}-2 \tilde{R})
$$

for $\tilde{R} \gg 1$ and up to an irrelevant additive term (Appendix A.1). The first term in Eq. (37) contributes a dominant (energetic) attractive force and the second term generates a repulsive force between the rods at small separations. Using Eq. (37), the rescaled equilibrium axial separation 


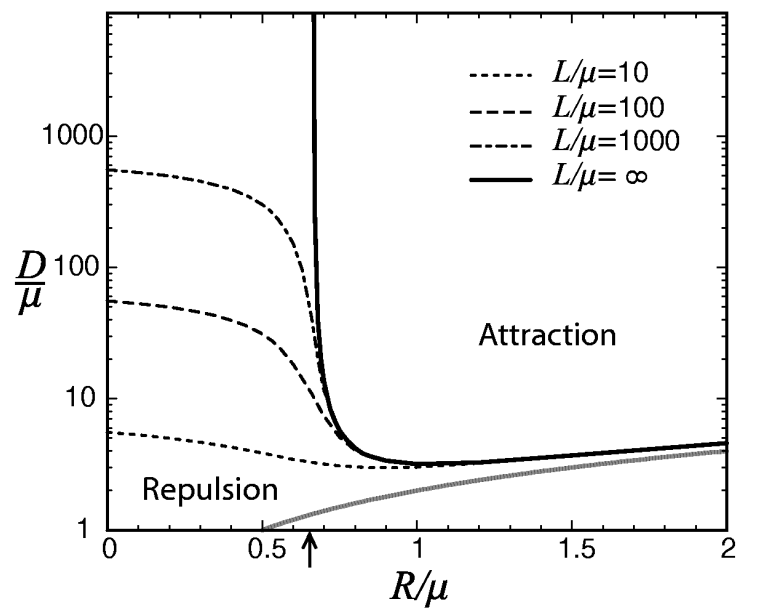

Fig. 4. Rescaled equilibrium axial separation of two likecharged rods plotted as a function of Manning parameter (the rescaled rod radius), $\tilde{R}=R / \mu$, for the box sizes $\tilde{L}=L / \mu=$ $10,100,1000, \infty$ as indicated on the graph. The small arrow locates the threshold Manning parameter $\tilde{R}_{c}=2 / 3$, and the thick grey curve corresponds to the contact axial separation $\tilde{D}_{\min }=2 \tilde{R}$.

is obtained approximately as

$$
\tilde{D}_{*} \approx 2 \tilde{R}+\frac{2}{3}+\mathcal{O}\left(\frac{1}{\tilde{R}}\right),
$$

for sufficiently large Manning parameter. Equation (38) shows that the (rescaled) equilibrium surface-to-surface distance of the rods, $\tilde{D}_{*}-2 \tilde{R}$, decreases and tends to a value of the order of unity as Manning parameter tends to infinity. Restoring the actual units in Eq. (38), we obtain $D_{*} \approx 2 R+2 \mu / 3+\mathcal{O}\left(\mu^{2} / R\right)$, where $R$ and $\mu$ are given by Eqs. (21) and (22). Thus, the actual equilibrium surfaceto-surface separation is obtained approximately as

$$
\Delta_{*} \equiv D_{*}-2 R_{0} \approx \sigma_{c}+\frac{2}{3} \mu+\mathcal{O}\left(\mu^{2}\right),
$$

when the Manning parameter is sufficiently large (or the Gouy-Chapman length, $\mu$, is small), that is about the counterion diameter.

For small Manning parameters $\tilde{R}<\tilde{R}_{c}=2 / 3$, qualitatively different features arise. The effective interaction between the rods exhibits a significant dependence upon the size of the confining box and the location of the minimum of the free energy tends to infinity for $\tilde{L} \rightarrow \infty$ resulting in a pure repulsion between unconfined rods (see Figure [3). This occurs as a result of the dilution of the counterion cloud around weakly-charged rods. For $\tilde{R} \ll 1$, the equilibrium axial separation scales with the box size and is obtained approximately as (Appendix A.1)

$$
\tilde{D}_{*} \approx \frac{\tilde{L}}{\sqrt{\pi}} .
$$

Now, for a given box size, the free energy varies smoothly from the typical forms shown in Figure 2 to those shown

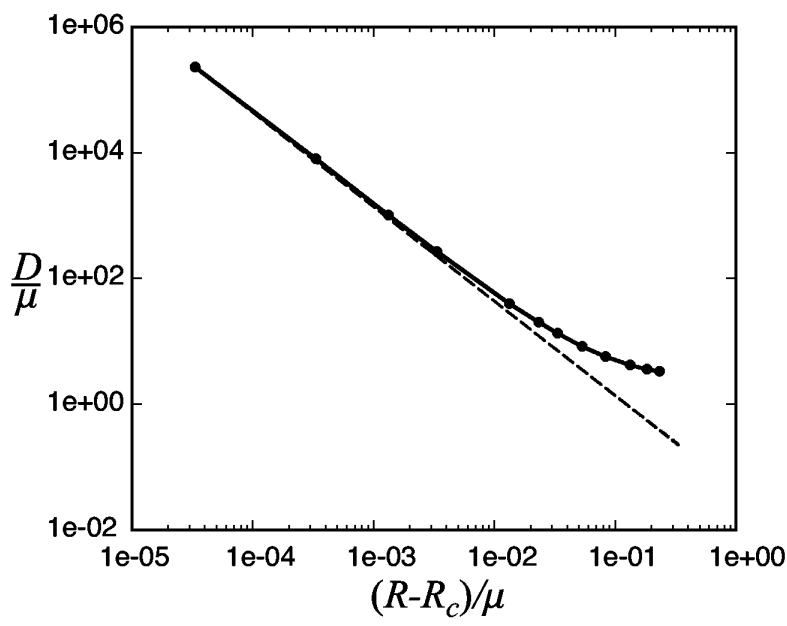

Fig. 5. Rescaled equilibrium axial separation of two unconfined rods $(\tilde{L}=\infty)$ in the vicinity of the threshold Manning parameter $R_{c}=2 / 3$ (solid curve with symbols). The dashed line shows a power-law fit with the slope $\alpha=3 / 2$ (see Eq. (41)).

in Figure 3 by decreasing Manning parameter. There is, however, a rapid change near $\tilde{R}_{c}=2 / 3$ indicative of a continuous unbinding transition in an infinitely large box. This is seen more clearly in Figure 4] where the predictions of the SC theory for the equilibrium axial separation is shown as a function of Manning parameter for various box sizes. (These results have been obtained directly by numerical minimization of the free energy, Eq. (27).) The region above a given curve corresponds to the axial separations at which the rods attract, and the region below that corresponds to the repulsion regime. When the box size tends to infinity, the equilibrium axial separation diverges for $\tilde{R} \rightarrow \tilde{R}_{c}^{+}$revealing the power-law behavior

$$
\tilde{D}_{*} \sim\left(\tilde{R}-\tilde{R}_{c}\right)^{-\alpha}
$$

shown in Figure 5. where within our numerical errors

$$
\alpha=3 / 2
$$

\subsection{The regime of applicability of SC results}

The system of two like-charged rods has been investigated in recent numerical simulations revealing strong attractive forces at moderate to large Manning parameters 13 23, 24,25]. The SC theory provides quantitative predictions for the equilibrium axial separation, that may be compared with simulations results. However, such comparison with realistic systems of finite coupling parameter (e.g. $\Xi \sim 10-100$ for simulations in Refs. [13,23, 24, 25] ) must be performed in a certain regime of parameters, where the present asymptotic theory (strictly valid only for $\Xi \rightarrow \infty$ ) is applicable. To this aim, extensive simulations have been performed recently by Arnold and 
Holm 24] covering a wide range of parameters, which allow for examining the influence of higher-order electrostatic and excluded-volume effects. The comparison with these results will be presented in separate publications [24], 25, and in the following, we merely introduce two criteria identifying the regime of parameters, where a reasonable agreement between simulations and SC predictions is obtained.

For highly-charged rods, excluded-volume interactions between counterions may be significant as attraction is accompanied by accumulation of counterions in a narrow region between the rods 24] (Appendix A.1). In this situation, the typical distance between intervening counterions (lining up in the $z$-direction), $a_{z}$, may be estimated from the local electroneutrality condition, $q=2 \tau a_{z}$, giving

$$
a_{z}=\frac{q}{2 \tau}
$$

where $q$ is the counterion valency and $\tau$ is the single-rod linear charge density. When, the counterion diameter, $\sigma_{c}$, is larger than $a_{z}$,

$$
a_{z}<\sigma_{c}
$$

the excluded-volume repulsions between counterions become important, and further accumulation of counterions between the rods according to the SC mechanism is prohibited. As a result, the equilibrium separation of the rods (determined from the electrostatic component of the total force between them) appears to be larger than the present predictions, since the strength of the mediated attraction drops. Though, it should be noted that volume interactions may indeed contribute an additional attractive component to the total force for highly-charged systems [23. 24. It is a priori not clear which of these effects is stronger.

On the other hand, as frequently quoted in the literature 16, 18, 19, 42, 47, 48, electrostatic correlations induced by counterions between two apposing macroions are dominant, when the typical distance between counterions, $a_{z}$, becomes larger than the macroions surface-to-surface distance, $\Delta=D-2 R_{0}$, i.e. when

$$
\Delta<a_{z}
$$

where $a_{z}$ is given by Eq. (43). In rescaled units, this condition reads

$$
\tilde{\Delta}<\frac{\Xi}{2 \xi}
$$

where the coupling parameter, Eq. (5), is

$$
\Xi=\frac{q^{3} \ell_{B}^{2} \tau}{R}
$$

for charged rods. The above criterion identifies the regime of parameters, where basically the counterion-rod correlations are superior to the counterion-counterion correlations and the present asymptotic theory is applicable 25 . 47. 48. For moderate coupling parameters $\Xi \sim 10$, this regime can be reached using charged rods with Manning parameter larger than $\xi \approx 1$ [25].

\section{Two Like-Charged Spheres}

Consider a system of two like-charged spheres each of radius $R_{0}$ and charge valency $Z$. The spheres are located at a center-to-center separation of $D$, and together with their neutralizing counterions are confined in a cubic box of edge size $L$. (The geometry of the system is similar to what we have sketched in Figure 1 where the two solid circles now display the largest cross-section of the spheres.) We assume the electric charge to be distributed uniformly over the surface of the spheres, thus their surface charge density reads $\sigma_{s}=Z /\left(4 \pi R^{2}\right)$, and the Gouy-Chapman length, Eq. (2), is

$$
\mu=\frac{2 R^{2}}{\ell_{B} q Z}
$$

where $R$ is the (hard-core) radius of spheres, Eq. (21). In rescaled units, we have

$$
\tilde{R}=\frac{R}{\mu}=\frac{\ell_{B} q Z}{2 R}
$$

which is referred to in the following as Manning parameter for charged spheres in analogy with the cylindrical case, Eq. (23).

To study the strong-coupling interaction of the two spheres, we follow similar lines as presented in the preceding Section by calculating the rescaled SC free energy, Eq. (19). The zero-particle and one-particle interaction energies are obtained from Eqs. (10) and (11) respectively as

$$
\begin{aligned}
\tilde{U}_{0} & =-28 \pi \frac{\tilde{R}^{4}}{\tilde{D}}, \\
\tilde{u}(\tilde{x}, \tilde{y}, \tilde{z}) & =-2 \tilde{R}^{2}\left[\frac{1}{\tilde{r}_{1}}+\frac{1}{\tilde{r}_{2}}-\frac{4}{\tilde{D}}\right],
\end{aligned}
$$

where

$$
\begin{aligned}
& \tilde{r}_{1}=\left[(\tilde{x}+\tilde{D} / 2)^{2}+\tilde{y}^{2}+\tilde{z}^{2}\right]^{1 / 2}, \\
& \tilde{r}_{2}=\left[(\tilde{x}-\tilde{D} / 2)^{2}+\tilde{y}^{2}+\tilde{z}^{2}\right]^{1 / 2},
\end{aligned}
$$

are radial distances from the centers of the spheres. The reference point is arbitrarily chosen to be in the mid-way between the spheres $\mathbf{r}_{0}=\mathbf{0}$, and self-energy terms are neglected. Using Eqs. (19) and (50), the rescaled SC free energy is obtained as

$$
\mathcal{F}_{1}=4 \frac{\tilde{R}^{4}}{\tilde{D}}-4 \tilde{R}^{2} \ln I
$$

where

$$
I(\tilde{D}, \tilde{R}, \tilde{L}) \equiv \int d \tilde{x} d \tilde{y} d \tilde{z} \tilde{\Omega} e^{2 \tilde{R}^{2}\left[\frac{1}{\tilde{r}_{1}}+\frac{1}{\tilde{r}_{2}}\right]}
$$

The geometry function $\tilde{\Omega}(\tilde{x}, \tilde{y}, \tilde{z} ; \tilde{D}, \tilde{R}, \tilde{L})$ specifies the region accessible for counterions, i.e. it is one inside the volume surrounded by the cubic box and the two spheres, and is zero elsewhere. 

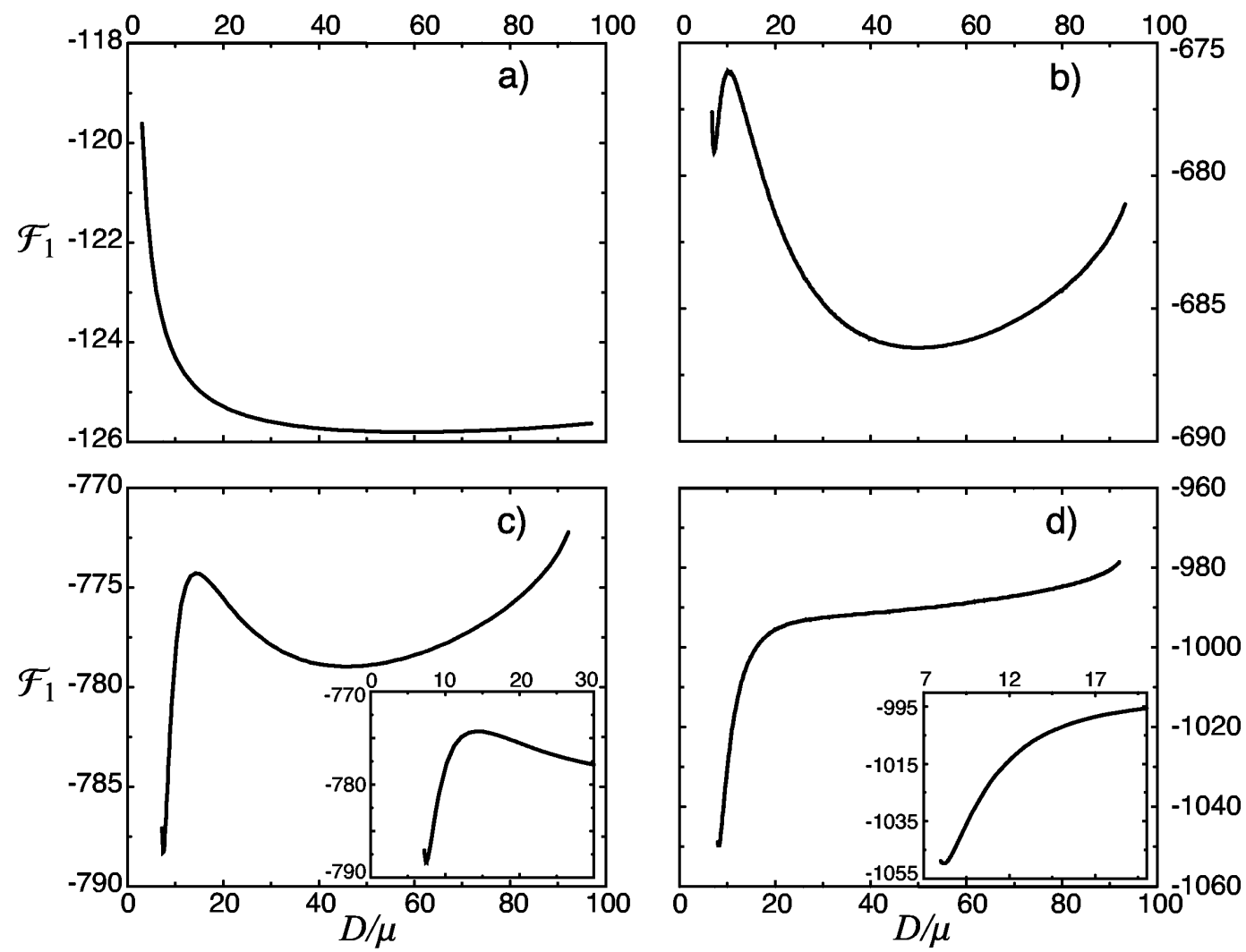

Fig. 6. The rescaled SC free energy of the two-sphere system, Eq. (52), plotted as a function of the rescaled center-to-center distance for different Manning parameters a) $\tilde{R}=1.5$, b) $\tilde{R}=3.4$, c) $\tilde{R}=3.6$ and d) $\tilde{R}=4.0$. The size of the confining box is $\tilde{L}=100$ in rescaled units. The insets show a closer view of the local minimum at $\tilde{D} \approx 2 \tilde{R}$.

The first term in Eq. (521) gives the long-ranged bare repulsion between the spheres, and the second term involving the spatial integral, $I$, contains energetic and entropic contributions of counterions on the leading order reproducing the counterion-condensation process and an attractive force for highly-charged spheres.

It follows easily from Eqs. (52) and (53) that for finite Manning parameter, the counterion-mediated force vanishes as the size of the confining box tends to infinity resulting in pure repulsion between unconfined spheres. This goes back to the fact that in the absence of confining boundaries, spherical macroions can not bind counterions that tend to diffuse in order to gain entropy. This is reflected by the divergency of the counterionic integral, Eq. (53), as $\tilde{L} \rightarrow \infty$. (Note that the involved integrand is always positive and larger than one, and upon rescaling the spatial coordinates as $\tilde{\mathbf{r}}^{\prime}=\tilde{\mathbf{r}} / \tilde{L}$, it scales with the volume of the confining box as $I \sim \tilde{L}^{3}$.) As a result, the distribution function of counterions [63] as well as the counterionmediated force,

$$
\tilde{F}_{c i} \sim \frac{\partial}{\partial \tilde{D}} \ln I
$$

that scales with the box size as $\sim \tilde{L}^{-2}$, vanish for increasing box size. In contrast, it turns out that two like-charged spheres can attract each other in any finite box provided that their Manning parameter is sufficiently large as will be demonstrated below.

Figures $6 \mathrm{a}-\mathrm{d}$ show the typical form of the SC free energy, calculated numerically (using Monte-Carlo integration methods) from Eq. (52), for two spheres in a box with $\tilde{L}=100$ and for several Manning parameters. As seen, at sufficiently small Manning parameter, the long-ranged repulsion is dominant and only a weak attraction operates, when the spheres are at large separations comparable with the box size (Figure 6 a). But at Manning parameters larger than a threshold $\tilde{R}_{c}^{(1)}$ (which is obtained as $\tilde{R}_{c}^{(1)} \approx$ 3.3 for $\tilde{L}=100$ ), a local minimum (short-ranged attraction) emerges at small separations about $\tilde{D} \approx 2 \tilde{R}$ corresponding to a meta-stable bound state of spheres (Figure [6]). The attractive interaction develops further and the potential barrier disappears, when the Manning parameter becomes larger than the second threshold $\tilde{R}_{c}^{(2)}$ (which is $\tilde{R}_{c}^{(2)} \approx 3.8$ for $\tilde{L}=100$ ) - see Figure [6. This clearly demonstrates the occurrence of a discontinuous transition between a closely-packed bound state and a repulsiondominated state of two spheres by changing Manning parameter $\tilde{R}$.

In Figure 7 we show the locations of the minima (solid curves) and the maximum (dashed curve) of the SC free energy as a function of Manning parameter for $\tilde{L}=100$ 


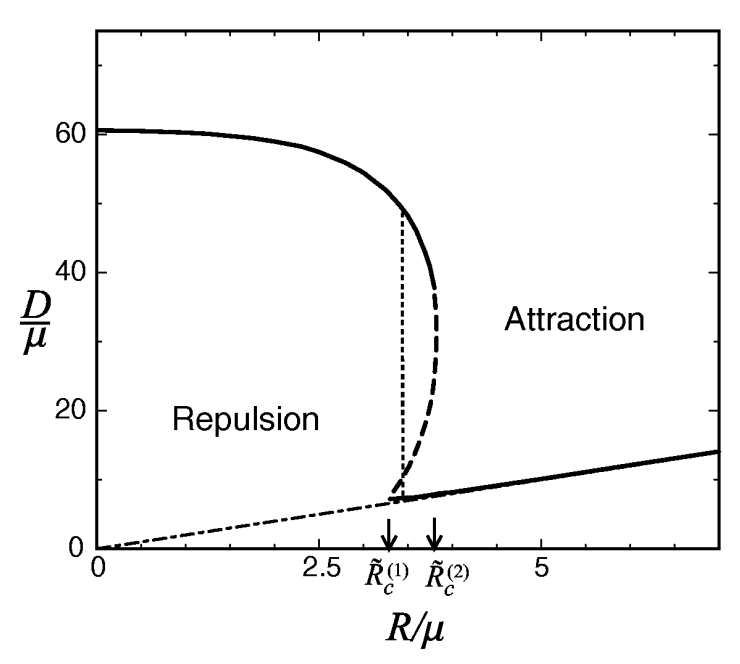

Fig. 7. Locations of the minima (solid curves) and the maximum (dashed curve) of the SC free energy for the two-sphere system plotted as a function of Manning parameter $\tilde{R}$ for $\tilde{L}=100$. The two arrows on the horizontal axis show the locations of the threshold Manning parameters $\tilde{R}_{c}^{(1)}$ and $\tilde{R}_{c}^{(2)}$. The dot-dashed line represents the contact separation $\tilde{D}_{\min }=2 \tilde{R}$, and the vertical dotted line shows the Manning parameter for which the values of the two minima of the free energy are equal.

(obtained numerically using Eq. (52)). The upper branch represents the shallow repulsion-dominated minimum, which is strongly sensitive to the box size. For small Manning parameter $\tilde{R} \ll 1$, the corresponding equilibrium centerto-center distance increases linearly with the box size and is obtained approximately as

$$
\tilde{D}_{*} \approx \sqrt[3]{\frac{3}{4 \pi}} \tilde{L}
$$

for $\tilde{L} \rightarrow \infty$ (Appendix A.2). By contrast, the small-separatio minimum (the lower branch) is effectively independent of the box size and is maintained essentially by an attractive interaction mediated by counterions intervening between the spheres. For sufficiently large Manning parameter $\tilde{R} \gg 1$, the approximate form of the rescaled $\mathrm{SC}$ free energy around its minimum at small separations is obtained as

$$
\mathcal{F}_{1}=-28 \frac{\tilde{R}^{4}}{\tilde{D}}-4 \tilde{R}^{2} \ln (\tilde{D}-2 \tilde{R})
$$

up to an unimportant additive term (Appendix A.2). For increasing Manning parameter (or decreasing temperature), the free energy expression (56) leads to an (energetic) attractive force independent of temperature, which scales with the center-to-center distance of the spheres, $D$, as $\sim 1 / D^{2}$, in agreement with results obtained by Shklovskii [45]. Using Eq. (56), the rescaled equilibrium center-to-center distance is obtained approximately as

$$
\tilde{D}_{*} \approx 2 \tilde{R}+\frac{4}{7}+\mathcal{O}\left(\frac{1}{\tilde{R}}\right)
$$

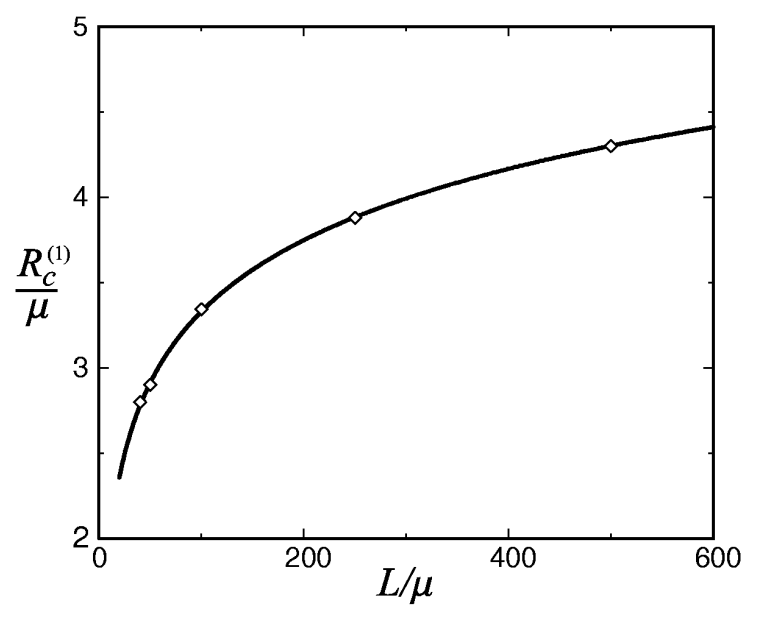

Fig. 8. The threshold Manning parameter $\tilde{R}_{c}^{(1)}$ plotted as a function of the box size $\tilde{L}$ (see Eq. (59) in the text). This threshold is obtained for box sizes larger than $\tilde{L} \approx 20$.

which yields an equilibrium surface-to-surface distance, $\tilde{D}_{*}-2 \tilde{R}$, of the order of unity in rescaled units. Now restoring the actual units in Eq. (57), we obtain $D_{*} \approx$ $2 R+4 \mu / 7+\mathcal{O}\left(\mu^{2} / R\right)$, where $R$ and $\mu$ are given by Eqs. (21) and (48). Thus, the actual equilibrium surface-tosurface separation of spheres is obtained approximately as

$$
\Delta_{*} \equiv D_{*}-2 R_{0} \approx \sigma_{c}+\frac{4}{7} \mu+\mathcal{O}\left(\mu^{2}\right),
$$

when the Manning parameter is sufficiently large (or the Gouy-Chapman length, $\mu$, is small), that is about the counterion diameter.

Similar features are obtained for box sizes that are larger than roughly $\tilde{L} \approx 20$; for smaller box size only the shallow large-distance minimum is obtained as depicted in Figure [6. The striking result is that the threshold Manhing parameters $\tilde{R}_{c}^{(1)}$ and $\tilde{R}_{c}^{(2)}$ monotonically increase by increasing the confinement volume. This behavior is shown in Figure 8 for $\tilde{R}_{c}^{(1)}$ (symbols), along with the best logarithmic fit to these results (solid curve) as

$$
\tilde{R}_{c}^{(1)}=a+b \ln \tilde{L},
$$

where $a \approx 0.55$ and $b \approx 0.6$. Such a weak increase of the attraction threshold demonstrates that even in a very large confinement, two like-charged spheres can fall into the attraction regime provided that the corresponding Manning parameter exceeds a moderate threshold. This behavior may also explain the stability of closely-packed clusters of highly-charged spheres in large confinement, and their insensitivity to the box size as addressed in recent simulations [14, 16, 18, 19]. The simulation parameters, where strong attraction has been observed, indeed covers the predicted attraction-dominated regime $\tilde{R} \gg \tilde{R}_{c}^{(1)}$ (see Table (1).

One should note that the unbinding transition can occur also by changing the confinement volume for a given Manning parameter. This is implied by the fact that, for 


\begin{tabular}{|c|c|c|c|c|c|c|c|c|c|c|c|c|c|}
\hline Simulation & $q$ & $Z$ & $\ell_{B}(\AA)$ & $R_{0}(\AA)$ & $\sigma_{c}(\AA)$ & $L(\AA)$ & $\mu(\AA)$ & $\tilde{R}$ & $\Xi$ & $\tilde{R}_{c}^{(1)}$ & $a_{\perp}(\AA)$ & $\Delta_{\operatorname{sim}}(\AA)$ & $\Delta_{*}(\AA)$ \\
\hline $\begin{array}{l}\text { Grønbech-Jensen } \\
\text { et al. 14] }\end{array}$ & 2 & 10 & 7.01 & 7 & 3.3 & $50-200$ & 1.07 & 8.1 & 26 & $2.8-3.7$ & 7.7 & 2.5 & $\overline{3.41}$ \\
\hline Wu et al. 15 & 2 & 20 & 7.14 & 10 & 4 & 100 & 1.01 & 11.9 & 28 & 3.3 & 7.5 & 4 & 4.10 \\
\hline $\begin{array}{l}\text { Allahyarov } \\
\text { et al. [16] }\end{array}$ & 2 & 32 & 112 & 48.9 & 4.4 & $\sim 10^{2}$ & 0.73 & 70.1 & 615 & $\sim 3.3$ & 25.5 & - & 4.41 \\
\hline Linse et al. $\begin{array}{lll}18 & 19\end{array}$ & 3 & 60 & 7.15 & 20 & 4 & $\sim 10^{2}$ & 0.75 & 29.2 & 85 & $\sim 3.3$ & 9.8 & 4 & 4.05 \\
\hline Hribar et al. 20 & 3 & 12 & 7.15 & 10 & 2 & $\sim 10^{2}$ & 0.94 & 11.7 & 68 & $\sim 3.3$ & 11.0 & - & 2.09 \\
\hline
\end{tabular}

Table 1. Parameters from simulations on highly-charged spheres (see the text for the definitions). The last two columns show the equilibrium surface-to-surface distance, $\Delta_{\text {sim }}$, obtained in these simulations (if explicitly measured), and the strong-coupling prediction, $\Delta_{*}=D_{*}-2 R_{0}$. Some of the numbers are given up to the order of magnitude, and the extracted values of $\Delta$ from simulations have a typical resolution of about $1 \AA$.

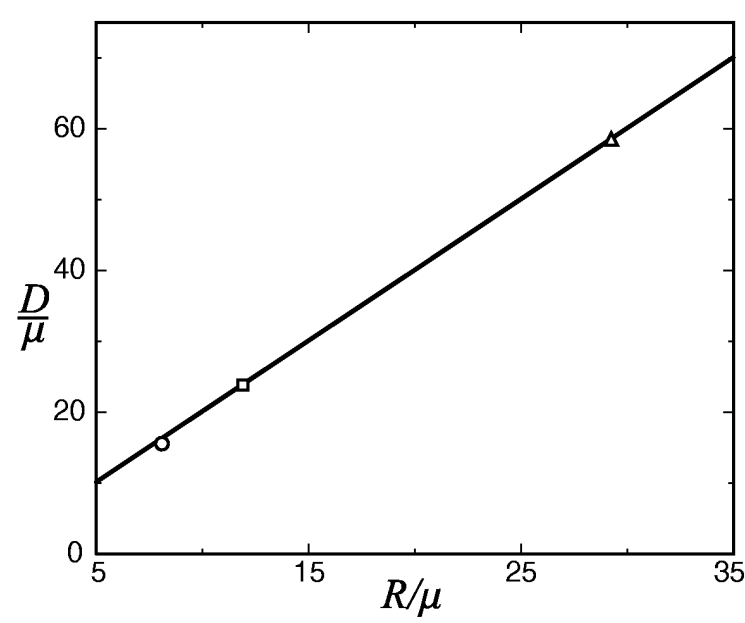

Fig. 9. Rescaled equilibrium center-to-center separation of like-charged spheres as a function of Manning parameter in the attraction-dominated regime (i.e. $\tilde{R} \gg \tilde{R}_{c}^{(1)}$ ). Symbols show data obtained from recent simulations (circle: Grønbech-Jensen et al. [14, square: Wu et al. [15, triangle: Linse et al. [18 19]) compared with the SC prediction (solid line).

a given Manning parameter between the two thresholds $\tilde{R}_{c}^{(1)}<\tilde{R}<\tilde{R}_{c}^{(2)}$, the height of the free energy barrier decreases slowly by increasing the box size. Qualitatively similar features have been obtained in the simulations by Grønbech-Jensen et al. 14] on a system of two like-charged spheres with divalent counterions. The obtained potential of mean force between spheres was shown to have a local minimum at small separation (the compact state) separated by a potential barrier from a repulsion regime (see Fig. 1 in Ref. 14, which schematically resembles Figure 6 . Note that the tail of the effective interaction is influenced by the boundary conditions, that is periodic in the case of the simulations). The barrier height was found to decrease for increasing confinement volume resulting in repulsion between spheres (Fig. 3 in Ref. 14]). The appearance of a potential barrier between two highly-coupled spheres was also noted in Ref. [21].

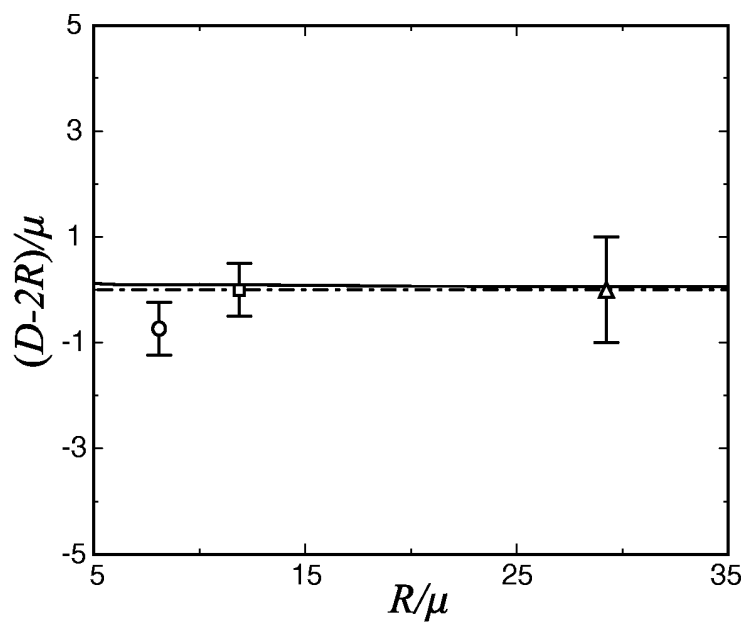

Fig. 10. Rescaled equilibrium surface-to-surface separation of like-charged spheres as a function of Manning parameter in the attraction-dominated regime. Symbols show data from recent simulations (circle: Grønbech-Jensen et al. 14, square: Wu et al. [15], triangle: Linse et al. [18, 19]) and the solid line is the $\mathrm{SC}$ prediction. Dot-dashed line shows the contact separation $\tilde{D}_{\min }=2 \tilde{R}$

The strong-coupling regime has also been investigated in several other simulations both on many-sphere systems [18, 19, 20 and on a single pair of spheres 15,16. Table 1 presents typical parameters from some of recent simulations. In the two last columns of the Table, we compare the equilibrium surface-to-surface separation between attracting spheres from these simulations, $\Delta_{\text {sim }}$ (if explicitly estimated), with the SC prediction, $\Delta_{*}=D_{*}-2 R_{0}$, in actual units. (In Figures 9 and [10] we also show the center-to-center distance, $\tilde{D}_{*}$, and the surface-to-surface distance, $\tilde{D}_{*}-2 \tilde{R}=\left(D_{*}-2 R_{0}-\sigma_{c}\right) / \mu$, in rescaled units. $)$ The strong-coupling results have been calculated numerically from the free energy, Eq. (52), and involve the finite size of counterions, which as discussed in Section 2.1 enters at the leading order via a finite closest approach distance between spheres and counterions. As seen, there is a qualitative agreement between the theoretical predictions 
and the simulations results. In fact, as addressed in previous numerical studies (see for example the Discussion in Ref. [19]), the equilibrium surface-to-surface separation of spheres in these simulations appears to be of the order of the counterion diameter. This result agrees with the SC prediction for highly-charged spheres of small GouyChapman length $(\mu \sim 1 \AA)$ as demonstrated by the limiting expression (58).

The regime of applicability of SC predictions to systems with finite coupling strength, such as the above simulations, may be examined by considering the ratio of the lateral counterion separation at charged spheres, $a_{\perp}$, to the surface-to-surface distance between the spheres, $\Delta=D-2 R_{0}$ [16, 18, 19, 42,47,48. The typical value of $a_{\perp}$ may be estimated from the local electroneutrality condition $\pi a_{\perp}^{2} \sim q / \sigma_{s}$, which holds up to a geometric factor of the order of one. In rescaled units, we get $a_{\perp} / \mu=\tilde{a}_{\perp} \sim$ $(2 \Xi)^{1 / 2}$, where the coupling parameter, Eq. (5), reads

$$
\Xi=\frac{q^{3} \ell_{B}^{2} Z}{2 R^{2}}
$$

for charged spheres. The lateral separation between counterions may also be written as

$$
a_{\perp} \sim R \sqrt{\frac{4 q}{Z}} .
$$

As discussed before, the SC regime, i.e. when higher-order corrections are small (Section [2), is characterized by the condition

$$
\Delta<a_{\perp}
$$

The estimated values of $a_{\perp}$ for the given simulations are shown in Table 1 As seen, the above criterion is fulfilled for the simulation parameters $16,18,19]$. In addition, the size of counterions are typically smaller than their lateral separation indicating that excluded-volume repulsion between counterions, which enters through higher-order corrections to the SC theory, is not a dominant effect.

\section{Conclusion and Discussion}

In this paper, we have presented the predictions of the strong-coupling theory for the effective interaction between two like-charged rods and two like-charged spheres. The SC theory obtained from a systematic virial expansion (Section 2) provides a framework to study strongly-coupled systems $(\Xi \gg 1)$. For large Manning parameters (or weaklycurved macroion surfaces), counterions effectively condense around macroions and a long-ranged attraction is obtained. (For charged rods, attraction is obtained even in an infinitely large confining box due to a universal counterioncondensation effect in this limit, while spheres show attraction only when they are confined in an outer box.) In general, the SC attraction is accompanied by strong accumulation of counterions in the intervening region between macroions that gives rise to attractive forces, which scale with the macroions separation, $D$, as $\sim 1 / D$ for two rods, and as $\sim 1 / D^{2}$ for two spheres. The equilibrium surface-to-surface separation of highly-charged macroions is then predicted to be of the order of the counterion diameter plus a term of the order of the Gouy-Chapman length - see Eqs. (39) and (58)- which is in qualitative agreement with results obtained from recent simulations on both charged rods 24] and charged spheres (Section 4). For point-like counterions, a quantitative agreement has been established with recent Molecular Dynamics simulations 25]. Some of the features such as the finite-size dependence of the effective interaction and the appearance of a potential barrier in a system of charged spheres are also consistent with the simulation results [14,21].

Note that in the simulations, attractive interactions are found to have a short-ranged character, whereas the SC theory predicts long-ranged attractions for large Manning parameters. This difference should be attributed to finite-coupling effects as in the simulations considered here 14, 15, 16, 18, 19,20,23, 24, 25], the coupling parameter is only moderately large, $\Xi \sim 10-10^{2}$. As it was demonstrated for planar charged walls [47.48, for such coupling parameters, the strength of electrostatic correlations is substantially decreased when charged walls are far from each other and consequently, the range of attraction is decreased. (In that case, it was shown that the largedistance behavior of the effective interaction is described by the PB theory and is purely repulsive). Yet, the SC results were shown to remain valid at sufficiently small separations, as obtained from the criterion explained in the text [25,47.48] (see Eqs. (45) and (62). As we already discussed, this criterion can be satisfied at moderate to large couplings using moderate values of Manning parameter, e.g. $\tilde{R}>1$ for two charged rods 25. Experimentally, this regime can be reached using multivalent counterions and highly-charged macroions. For instance, in aqueous solutions of DNA (with radius $R_{0} \approx 10 \AA$ and linear charge density $\tau e \approx 6 e / \mathrm{nm}$ ), Manning parameter and the coupling parameter are as large as $\tilde{R} \approx 8$ and $\Xi \approx 25$ in the presence of divalent counterions, and as $\tilde{R} \approx 12$ and $\Xi \approx 80$ for trivalent counterions such as spermidine. In colloidal dispersions, an aqueous solution of highly-charged surfactant micelles of, for example, typical radius $R_{0} \approx 20 \AA$ and charge valency $Z \approx 60$ represents Manning parameter and the coupling parameter of the order of $\widetilde{R} \approx 30$ and $\Xi \approx 100$ for trivalent counterions. It is also noted that recent experimental observation of attraction between like-charged colloidal particles in Refs. 64 65 66 concerns the regime of small coupling parameters (typically with $\Xi \sim 10^{-2}-10^{-1}$ due to the large size of spheres $\sim 1 \mu \mathrm{m})$. These observations, therefore, should not be compared with the strong-coupling results.

We have also examined the predictions of the SC theory for small Manning parameter (or small radius of curvature), e.g. for the onset of attraction. For sufficiently small Manning parameters and increasing confinement volume, one expects that counterions completely de-condense leading to pure repulsion between macroions. It should be noted, however, that when counterions de-condense, counterionmacroion correlations become effectively small and contributions from higher-order corrections to the asymptotic 
theory may become important even for large coupling parameters (this can be understood also in terms of the criterion introduced in Sections 3.3 and 4). These effects might result in finite corrections for the predicted threshold of attraction or scaling exponents, such as Eq. (42), in the limit of large couplings. However, as we showed, the decondensation process at small Manning parameters is captured by the SC free energy and a dominant repulsive interaction is obtained, which gives a consistent picture for the whole range of Manning parameters. Note also that the threshold of condensation obtained for two charged rods (Section 3.1) coincides with the value obtained from Manning theory 49,59]. Further numerical studies in this regime will be useful to investigate the role of higher-order corrections. (These aspects have been studied for a singlerod system by means of Monte-Carlo simulations [60.)

Finally, we emphasize that the thermodynamic behavior of macroionic solutions and colloidal dispersions may not be derived directly from the SC free energy presented in Sections 3 and 4 As we showed, by decreasing Manning parameter or increasing the box size, attracting macroions undergo an unbinding transition to a repulsion-dominated state, which occurs continuously for two rods and discontinuously for two spheres. These transitions do not necessarily represent thermodynamic phase transitions, because in our study, the distance between macroions is assumed to be fixed, while in realistic situations (as well as in the simulations on charged spheres cited in Section 4), the macroion-macroion separation is an annealed degree of freedom contributing separately to the partition function. Also, for fluctuating spheres there will be a logarithmic contribution to the entropy for large separations due to the increasing free volume available for the sphere-sphere distance coordinate. On the other hand, there has been evidence of a thermodynamic phase transition of the first order (phase separation) from recent numerical simulations on highly-charged spheres [14,18, 19,20]. The systematic study of such phase transitions in the strong-coupling limit will be subject of a future investigation.

We are grateful to H. Boroudjerdi, R. Golestanian, A.G. Moreira and R. Podgornik for useful discussions, and to A. Arnold and C. Holm for providing us with their recent numerical results on the two-rod system. We acknowledge financial supports from DFG Schwerpunkt Polyelektrolytes with defined architecture and DFG German-French Network.

\section{A Asymptotic analysis of the SC free energy}

\section{A.1 The two-rod system}

Let us first consider the limit of vanishingly small Manning parameter $\tilde{R} \ll 1$. In this case, the integral in Eq. (27) may be expanded in terms of $\tilde{R}$, and the rescaled SC free energy per unit length, $\tilde{H}$, of the rods is subsequently obtained as

$$
\frac{\mathcal{F}_{1}}{\tilde{H}} \approx-2 \tilde{R}^{2} \ln \tilde{D}+\frac{4 \tilde{R}^{2}}{\tilde{L}^{2}} \int_{-\tilde{L} / 2}^{\tilde{L} / 2} d \tilde{x} d \tilde{y}\left[\ln \tilde{r}_{1}+\ln \tilde{r}_{2}\right]
$$

$$
-2 \tilde{R} \ln \tilde{L}^{2}
$$

where we have omitted terms that are independent of $\tilde{L}$ and $\tilde{D}$ as they are irrelevant in our discussion. The asymptotic expression (63) involves the rescaled mean energy of the system of two-rods and neutralizing counterions (the first two terms) together with the entropic contribution of the counterions (the last term), which has an ideal-gas form. (This is seen more clearly using Eqs. (20) and (63) and restoring the actual units that yields $\mathcal{F}_{N}^{S C} \approx-2 \ell_{B} \tau^{2} H \ln D+2 \xi N\left\langle\ln r_{1}+\ln r_{2}\right\rangle-N \ln L^{2}$ per $k_{B} T$ and up to some additive terms. Note that Manning parameter equals the rescaled radius of rods, Eq. (23), thus $\tilde{R} \rightarrow 0$ implies line charges in the rescaled picture.) This form of the SC free energy shows that at small Manning parameters, the counterions are effectively unbound, though still interacting with the charged rods. The bare repulsive force between the rods coming from the first term scales like $\sim \tilde{D}^{-1}$, but the counterion-induced attraction force coming from the second term scales as $\sim \tilde{D} / \tilde{L}^{2}$, which becomes vanishingly small as $\tilde{L} \rightarrow \infty$. Therefore, the asymptotic free energy (63) admits a shallow $\tilde{L}$-dependent minimum as it is seen in Figure 3. By minimizing expression (63) with respect to $\tilde{D}$, the boundstate separation is approximately found as in Eq. (40) for $\tilde{L} \rightarrow \infty$.

For large $\tilde{R}>\tilde{R}_{c}$, as seen in Figure 2 the free energy substantially decreases, when the rods are close to each other. Inspection shows that in this situation, the main contribution to the integral $I$ in Eq. (27) comes from the intervening region between the rods $(-\tilde{D} / 2+\tilde{R}<\tilde{x}<$ $\tilde{D} / 2-\tilde{R} ; \tilde{y} \approx 0)$. In fact, this is associated with strong accumulation of counterions in this region for increasing Manning parameter, as it can be checked directly from the counterionic distribution function obtained in the SC limit 63. The integral I, Eq. (28), may be rewritten as

$$
I=\int d \tilde{x} d \tilde{y} \tilde{\Omega} e^{-2 \tilde{R} g(\tilde{x}, \tilde{y})}
$$

where

$$
g(\tilde{x}, \tilde{y})=\left[\ln \tilde{r}_{1}+\ln \tilde{r}_{2}\right],
$$

and $\tilde{r}_{1,2}$ are defined in Eq. (26). It turns out that $(\tilde{x}, \tilde{y})=$ $(0,0)$ is the saddle point of $g$, thus for sufficiently large $\tilde{R}$, we may use a saddle-point approximation to calculate $I$, which gives (up to some irrelevant prefactors)

$$
I \approx e^{-2 \tilde{R} \ln \tilde{D}^{2}} \times \tilde{D}^{2} \times \int_{-1 / 2+\tilde{R} / \tilde{D}}^{1 / 2-\tilde{R} / \tilde{D}} d \tilde{x} e^{8 \tilde{R} \tilde{x}^{2}}
$$

where we have rescaled the coordinates as $\tilde{x} \rightarrow \tilde{x} / \tilde{D}, \tilde{y} \rightarrow$ $\tilde{y} / \tilde{D}$ (Eq. (30) and assumed that the box size is sufficiently large. Indeed, the above approximation is valid only for sufficiently large $\tilde{R} / \tilde{D}$ ratio, since $g$ is singular at $(\tilde{x} / \tilde{D}, \tilde{y} / \tilde{D})=( \pm 1 / 2,0)$. In other words, it remains valid as long as the surface-to-surface separation of rods is sufficiently small, i.e. $\delta=(\tilde{D}-2 \tilde{R}) / 2 \tilde{R} \ll 1$. In this situation, the following approximate expression is obtained 
for the rescaled free energy using Eqs. (66) and (27),

$$
\frac{\mathcal{F}_{1}}{\tilde{H}} \approx 6 \tilde{R}^{2} \ln \tilde{D}-2 \tilde{R} f(\tilde{D}, \tilde{R})
$$

where $f \approx \ln (\tilde{D}-2 \tilde{R})+\mathcal{O}(\delta)$. Now, by minimizing expression (67) with respect to $\tilde{D}$, the equilibrium axial separation is obtained as in Eq. (38) for $\tilde{R} \gg 1$. We emphasize that Eq. (67) represents the approximate form of the free energy around its local minimum, and is not valid for large separations (or small Manning parameters close to the attraction threshold $\tilde{R}_{c}=2 / 3$ ). The large-distance behavior of the free energy is given by Eqs. (33) and (34).

Restoring the actual units in Eq. [67), and noting that the rescaled free energy $\mathcal{F}_{1}$ is related to the actual SC free energy (per $\left.k_{B} T\right), \mathcal{F}_{N}^{S C}$, through Eq. (20), we find

$$
\mathcal{F}_{N}^{S C} \approx 6 \ell_{B} \tau^{2} H \ln D-N \ln (D-2 R) .
$$

The first term in this equation formally corresponds to the energetic attraction between two rods mediated by neutralizing counterions that are located between them (lining up in $z$ direction with an effective linear charge density of $2 \tau$ ). While the second term may be regarded as an (repulsive) entropic contribution from counterions. Note that the attractive component of the resultant force is independent of the temperature, which only appears as a prefactor in the second term. Clearly, for increasing Manning parameter (or decreasing temperature), the strength of attraction increases [13 34] and saturates to the maximal value given by the first term in Eq. (68).

\section{A.2 The two-sphere system}

For vanishingly small Manning parameter $\tilde{R} \ll 1$, one may expand the integral involved in the rescaled SC free energy, Eq. (521), which yields

$$
\begin{aligned}
\mathcal{F}_{1} & \approx 4 \frac{\tilde{R}^{4}}{\tilde{D}}-\frac{8 \tilde{R}^{4}}{\tilde{L}^{3}} \int_{-\tilde{L} / 2}^{\tilde{L} / 2} d^{3} \tilde{r}\left[\frac{1}{\tilde{r}_{1}}+\frac{1}{\tilde{r}_{2}}\right] \\
& -4 \tilde{R}^{2} \ln \tilde{L}^{3} .
\end{aligned}
$$

This expression is basically similar to the low-Manningparameter expansion for the two-rod system, Eq. (63), containing the mean energy of the two spheres with neutralizing counterions (the first two terms) together with the entropic contribution of the counterions (the last term) that has an ideal-gas form. (This is seen using Eqs. (20) and (69) and restoring the actual units that gives $\mathcal{F}_{N}^{S C} \approx$ $\ell_{B} Z^{2} / D-N \ell_{B} Z q\left\langle 1 / r_{1}+1 / r_{2}\right\rangle-N \ln L^{3}$ in units of $k_{B} T$.) The bare repulsive force coming from the first term scales like $\sim \tilde{D}^{-2}$, but the counterion-induced attraction force coming from the second term scales as $\sim \tilde{D} / \tilde{L}^{3}$, which becomes vanishingly small as $\tilde{L} \rightarrow \infty$. The asymptotic free energy (69) has a shallow $\tilde{L}$-dependent minimum as seen in Figures $[\mathrm{a}$-c, the location of which is approximately obtained as in Eq. (55) in the text.
For large Manning parameter, as seen in Figure 64, the free energy exhibits a local minimum at small center-tocenter separations, where the main contribution to the volume integral in Eq. (52) comes from the intervening region between spheres $(-\tilde{D} / 2+\tilde{R}<\tilde{x}<\tilde{D} / 2-\tilde{R} ; \tilde{y} \approx 0 ; \tilde{z} \approx 0)$. In this case, a saddle-point approximation similar to the two-rod system (Eq. [66) ) may be performed, which gives the following asymptotic expression for the rescaled free energy for sufficiently large $R$ and small surface-to-surface separation $\delta=(\tilde{D}-2 \tilde{R}) / 2 \tilde{R} \ll 1$,

$$
\mathcal{F}_{1}=-28 \frac{\tilde{R}^{4}}{\tilde{D}}-4 \tilde{R}^{2} f(\tilde{D}, \tilde{R}),
$$

where $f \approx \ln (\tilde{D}-2 \tilde{R})+\mathcal{O}(\delta)$. The equilibrium separation is subsequently obtained by minimizing expression (70) with respect to $\tilde{D}$ yielding Eq. (57) in the text.

Using the free energy expression (70) and restoring the actual units, the approximate form of the actual SC free energy, Eq. (20), is obtained as

$$
\mathcal{F}_{N}^{S C} \approx-7 \ell_{B} \frac{Z^{2}}{D}-N \ln (D-2 R) .
$$

The first term in Eq. (71) formally corresponds to the energetic attraction between two spheres mediated by a neutralizing counterion (of charge valency $q=2 Z$ ) located between them. The second terms may be regarded as an (repulsive) entropic contribution from counterions. Upon increasing Manning parameter (or decreasing the temperature), the strength of attraction increases and saturates to the maximal value given by the first term in Eq. (71), which gives a temperature-independent force that scales with the center-to-center distance as $\sim 1 / D^{2}$.

\section{References}

1. V.A. Bloomfield, Biopolymers 31, 1471 (1991); Curr. Opin. Struct. Biol. 6, 334 (1996).

2. M. Delsanti, J.P. Dalbiez, O. Spalla, L. Belloni, M. Drifford, ACS Symp. Ser. 548, 381 (1994).

3. P. Gonzalez-Monzuelos, M. Olvera de la Cruz, J. Chem. Phys. 103, 3145 (1995); M. Olvera de la Cruz, L. Belloni, M. Delsanti, J.P. Dalbiez, O. Spalla, M. Drifford, J. Chem. Phys. 103, 5781 (1995).

4. H.H. Strey, R. Podgornik, D.C. Rau, V.A. Parsegian, Curr. Opin. Struct. Biol. 8, 309 (1998).

5. J.X. Tang, S. Wong, P. Tran, P.A. Janmey, Ber. BunsenGes. Phys. Chem. 100, 1 (1996); J.X. Tang, T. Ito, T. Tao, P. Traub, P.A. Janmey, Biochemistry 36, 12600 (1997).

6. G.C.L. Wong, A. Lin, J.X. Tang, Y. Li, P.A. Janmey, C.R. Safinya, Phys. Rev. Lett. 91, 018103 (2003); J.C. Butler, T. Angelini, J.X. Tang, G.C.L. Wong, Phys. Rev. Lett. 91, 028301 (2003).

7. L. Guldbrand, B. Jönsson, H. Wennerström, P. Linse, J. Chem. Phys. 80, 2221 (1984).

8. B. Svensson, B. Jönsson, Chem. Phys. Lett. 108, 580 (1984).

9. L. Guldbrand, L.G. Nilsson, L. Nordenskiöld, J. Chem. Phys. 85, 6686 (1986); L.G. Nilsson, L. Guldbrand, L. Nordenskiöld, Mol. Phys. 72, 177 (1991). 
10. C.E. Woodward, B. Jönsson, T. Åkesson, J. Chem. Phys. 89, 5145 (1988).

11. J.P. Valleau, R. Ivkov, G.M. Torrie, J. Chem. Phys. 95, 520 (1991).

12. A.P. Lyubartsev, L. Nordenskiöld, J. Phys. Chem. 99, 10373 (1995).

13. N. Grønbech-Jensen, R.J. Mashl, R.F. Bruinsma, W.M. Gelbart, Phys. Rev. Lett. 78, 2477 (1997).

14. N. Grønbech-Jensen, K.M. Beardmore, P. Pincus, Physica A 261, 74 (1998).

15. J.W. Wu, D. Bratko, J.M. Prausnitz, Proc. Natl. Acad. Sci. USA 95, 15169 (1998).

16. E. Allahyarov, I. D’Amico, H. Löwen, Phys. Rev. Lett. 81, 1334 (1998).

17. M.J. Stevens, Phys. Rev. Lett. 82, 101 (1999).

18. P. Linse, V. Lobaskin, Phys. Rev. Lett. 83, 4208 (1999).

19. P. Linse, V. Lobaskin, J. Chem. Phys. 112, 3917 (2000); P. Linse, J. Chem. Phys. 113, 4359 (2000).

20. B. Hribar, V. Vlachy, Biophys. J. 78, 694 (2000).

21. R. Messina, C. Holm, K. Kremer, Phys. Rev. Lett. 85, 872 (2000); Europhys. Lett. 51, 461 (2000).

22. E. Allahyarov, H. Löwen, Phys. Rev. E 62, 5542 (2000).

23. M. Deserno, A. Arnold, C. Holm, Macromolecules 36, 249 (2003).

24. A. Arnold, C. Holm, in preparation.

25. A. Naji, A. Arnold, C. Holm, R.R. Netz, Europhys. Lett. 67, 130 (2004).

26. R. Kjellander, S. Marčelja, Chem. Phys. Lett. 112, 49 (1984); J. Chem. Phys. 82, 2122 (1985).

27. R. Kjellander, T. Åkesson, B. Jönsson, S. Marčelja, J. Chem. Phys. 97, 1424 (1992).

28. F. Oosawa, Biopolymers 6, 1633 (1968).

29. P. Attard, R. Kjellander, D.J. Mitchell, Chem. Phys. Lett. 139, 219 (1987); P. Attard, D.J. Mitchell, B.W. Ninham, J. Chem. Phys. 88, 4987 (1988); P. Attard, R. Kjellander, D.J. Mitchell, B. Jönsson, J. Chem. Phys. 89, 1664 (1988).

30. R. Podgornik, J. Phys. A: Math. Gen. 23, 275 (1990); R. Podgornik, B. Žekš, J. Chem. Soc., Faraday Trans. II 84, 611 (1988).

31. J.-L. Barrat, J.-F. Joanny, Adv. Chem. Phys. XCIV, 1 (1996).

32. P.A. Pincus, S.A. Safran, Europhys. Lett. 42, 103 (1998).

33. R. Podgornik and V.A. Parsegian, Phys. Rev. Lett. 80, 1560 (1998).

34. B.-Y. Ha, A.J. Liu, Phys. Rev. Lett. 79, 1289 (1997); Phys. Rev. Lett. 81, 1011 (1998); Phys. Rev. E 58, 6281 (1998); Phys. Rev. E 60, 803 (1999); Phys. Rev. Lett. 83, 2681 (1999).

35. D.B. Lukatsky, S.A. Safran, Phys. Rev. E 60, 5848 (1999).

36. M. Kardar, R. Golestanian, Rev. Mod. Phys. 71, 1233 (1999).

37. R.R. Netz, H. Orland, Eur. Phys. J. E 1, 203 (2000).

38. B.-Y. Ha, Phys. Rev. E 64, 031507 (2001).

39. A.W.C. Lau, P. Pincus, Phys. Rev. E 66, 041501 (2002).

40. M.J. Stevens, M.O. Robbins, Europhys. Lett. 12, 81 (1990).

41. A. Diehl, M.N. Tamashiro, M.C. Barbosa, Y. Levin, Physica A 274, 433 (1999); M.C. Barbosa, M. Deserno, C. Holm, Europhys. Lett. 52, 80 (2000).

42. I. Rouzina, V.A. Bloomfield, J. Phys. Chem. 100, 4292 (1996); J. Phys. Chem. 100, 9977 (1996).

43. A.A. Kornyshev, S. Leikin, J. Chem. Phys. 107, 3656 (1997); Phys. Rev. Lett. 82, 4138 (1999).
44. J.J. Arenzon, J.F. Stilck, Y. Levin, Eur. Phys. J. B 12, 79 (1999); Y. Levin, J.J. Arenzon, J.F. Stilck, Phys. Rev. Lett. 83, 2680 (1999); A. Diehl, H.A. Carmona, Y. Levin, Phys. Rev. E 64, 011804 (2001).

45. B.I. Shklovskii, Phys. Rev. Lett. 82, 3268 (1999); T.T. Nguyen, I. Rouzina, B.I. Shklovskii, J. Chem. Phys. 112, 2562 (2000).

46. A.W.C. Lau, D. Levine, P. Pincus, Phys. Rev. Lett. 84, 4116 (2000); A.W.C. Lau, P. Pincus, D. Levine, H.A. Fertig, Phys. Rev. E 63, 051604 (2001).

47. R.R. Netz, Eur. Phys. J. E 5, 557 (2001); A.G. Moreira, R.R. Netz, Europhys. Lett. 52, 705 (2000); Europhys. Lett. 57, 911 (2002).

48. A.G. Moreira, R.R. Netz, Phys. Rev. Lett 87, 078301 (2001); Eur. Phys. J. E 8, 33 (2002).

49. J. Ray, G.S. Manning, Macromolecules 30, 5739 (1997); Langmuir 10, 2450 (1994).

50. I. Rouzina, V.A. Bloomfield, Biophys. J. 74, 3152 (1998).

51. R. Golestanian, M. Kardar, T.B. Liverpool, Phys. Rev. Lett. 82, 4456 (1999).

52. Y. Levin, Physica A 265, 432 (1999); Rep. Prog. Phys. 65, 1577 (2002).

53. O. Spalla, L. Belloni, Phys. Rev. Lett. 74, 2515 (1995); L. Belloni, J. Phys.: Condens. Matter 12, R549 (2000).

54. E.J. Verwey, J.T.G. Overbeek, Theory of the stability of lyophobic colloids (Elsevier, Amsterdam, 1948).

55. P. Debye, E. Hückel, Physik. Z. 24, 185 (1923).

56. J.C. Neu, Phys. Rev. Lett. 82, 1072 (1999).

57. J.E. Sader, D.Y.C. Chan, J. Colloid Interface Sci. 213, 268 (1999); Langmuir 16, 324 (2000).

58. E. Trizac, J.-L. Raimbault, Phys. Rev. E 60, 6530 (1999); E. Trizac, Phys. Rev. E 62, R1465 (2000).

59. G.S. Manning, J. Chem. Phys. 51, 924 (1969).

60. A. Naji, R.R. Netz, to be published.

61. In general, the proposed expansion, Eq. (15), may also involve additional logarithmic terms 47.

62. As seen from Eq. [19], the rescaled SC free energy, $\mathcal{F}_{1}$, is independent of the coupling parameter, $\Xi$, thus $\mathcal{F}_{N}^{S C}$ is inversely proportional to $\Xi$. As a result, effective pressures (as well as other observables such as counterions density profile) calculated from $\mathcal{F}_{N}^{S C}$ will be independent of $\Xi$ on the leading order [47. For instance, note that the rescaled pressure follows from the formal relation $\tilde{P}=-2 \pi \Xi\left(\partial \mathcal{F}_{N} / \partial \tilde{V}\right)$, where $\tilde{V}$ has (rescaled) volume dimension.

63. In the strong-coupling limit $\Xi \rightarrow \infty$, the counterionic density profile around charged objects is obtained as $\tilde{\rho}(\tilde{\mathbf{r}})=$ $\Lambda_{0} \tilde{\Omega}(\tilde{\mathbf{r}}) \exp (-\tilde{u}(\tilde{\mathbf{r}}))$ 47, where $\Lambda_{0}$, defined in Eq. (16), is inversely proportional to $I$ defined in Eqs. (28) and (153) for two rods and two spheres respectively. When $I$ diverges, the density profile of counterions vanishes.

64. J.C. Crocker, D.G. Grier, Phys. Rev. Lett. 73, 352 (1994); Phys. Rev. Lett. 77, 1897 (1996).

65. G.M. Kepler, S. Fraden, Phys. Rev. Lett. 73, 356 (1994); M.D. Carbajal-Tinoco, F. Castro-Román, J.L. ArauzLara, Phys. Rev. E 53, 3745 (1996).

66. N. Ise, T. Okubo, M. Sugimura, K. Ito, H.J. Nolte, J. Chem. Phys. 78, 536 (1983); B.V.R. Tata, M. Rajalakshmi, A.K. Arora, Phys. Rev. Lett. 69, 3778 (1992); T. Palberg, M. Würth, Phys. Rev. Lett. 72, 786 (1994). 